\title{
PERTURBATIVE THEORY OF MICA POLYTYPISM. ROLE OF THE M2 LAYER IN THE FORMATION OF INHOMOGENEOUS POLYTYPES
}

\author{
Massimo Nespolo \\ Japan Science and Technology Corporation, National Institute for Research in Inorganic Materials, 1-1 Namiki, \\ Tsukuba-shi, Ibaraki 305-0044, Japan
}

\begin{abstract}
A new model is proposed to explain, within the framework of the theory of spiral growth of Frank, the formation on inhomogeneous mica polytypes. This model relates the interaction and cooperative growth of two components (spirals and/or crystals) to produce a new stacking sequence. Depending on the relative orientation between the two components, a mismatch of the interlayer positions occurs, which is compensated through either a growth defect or a crystallographic slip at the octahedral $(O)$ sheet. Both these adjustments transform the M1 layer into the M2 layer. These two types of layers have the same chemical composition but differ in cation distribution in the $O$ sheet. The coalescence and cooperative growth of crystals occurs in fluid-rich environments and is most frequent in druses and volcanic fumaroles. These environments favor the inhomogeneous polytypes, especially those with complex stacking sequences. In addition, the $\mathrm{M} 1 \rightarrow \mathrm{M} 2$ transformation is most probable in micas with an oxybiotitic composition, where the removal of the $\mathrm{OH}$ dipole strengthens the interlayer bonding and the presence of high-charge cations destabilizes the $O$ sheet. Three examples of inhomogeneous polytypes of titaniferous oxybiotite from Ruiz Peak (a volcanic environment where many inhomogeneous polytypes have been reported) are presented.
\end{abstract}

Key Words-Crystal Growth, Long-Period Polytypes, Mica, Polytypism.

\section{INTRODUCTION}

Micas are phyllosilicates built by a $2: 1$ or $T-O-T$ layer, henceforth called "M layer", (Figures 1 and 2) with interlayer cations between adjacent layers. In the ideal structure (Pauling, 1930), the interlayer cations are in hexagonal prismatic coordination, with 12 nearest-neighbor oxygen atoms, six from each $M$ layer, and the layer symmetry is $P(6) \mathrm{mm}$ (layer-group notation after Dornberger-Schiff, 1959). The most relevant structural distortion occurring in the real layer is the rotation of the tetrahedra in the $T$ sheets about the normal to the layer, by an angle that depends upon crystal chemistry and on temperature and pressure (Takeda and Morosin, 1975; Catti et al., 1989). Consequently, the symmetry of the $T$ sheets is reduced to $P(3) 1 \mathrm{~m}$ and the 12 oxygen atoms around the interlayer cation separate into six nearest-neighbors and six nextnearest neighbors: The site hosting the interlayer cation becomes a trigonal (considering the nearest six oxygen atoms) or ditrigonal (considering all 12 oxygen atoms) antiprism for $2 n \times 60^{\circ}$ rotations of the layers, or a trigonal/ditrigonal prism for $(2 n+1) \times 60^{\circ}$ rotations ( $n$ integer, $0 \leq n \leq 5$ ). The latter coordination polyhedron is energetically less favorable, because the basal oxygen atoms are superimposed in the (001) projection.

In the interlayer region between two adjacent $M$ layers, monovalent and, less frequently, divalent cations occur. Polytypism arises from the stacking of the $\mathbf{M}$ layer along the $c$ axis: the geometrical operation relating pairs of layers is a two-fold axis in the interlayer, and adjacent layers result rotated by $n \times 60^{\circ}(0 \leq$ $n \leq 5)$ about $c^{*}$. Polytypes in which the position of any layer relative to the others and the transition from it to the adjacent ones are the same or equivalent for all layers are termed homogeneous (Zvyagin, 1988). Homogeneous polytypes have successive M layers rotated always by the same angle, apart from the sign, and correspond to the so-called "simple polymorphs" in Smith and Yoder (1956). By using Ramsdell (1947) notation, homogeneous polytypes are identified as $1 M$, $2 M_{1}, 3 T, 2 M_{2}, 2 O$, and $6 H$ (symbols for polytype symmetry according to Guinier et al., 1984). The remaining polytypes are termed inhomogeneous and correspond to the "complex polymorphs" in Smith and Yoder (1956). Mica polytypes are then suitably classified into three kinds, depending on the geometrical equivalence of layer pairs (Ďurovič et al., 1984; Nespolo, 1999): 1) subfamily-A polytypes: successive layers are rotated by $2 n \times 60^{\circ}$ only; 2) subfamily- $B$ polytypes: successive layers are rotated by $(2 n+1) \times$ $60^{\circ}$ only; 3) mixed-rotation polytypes: successive layers are rotated by both $2 n \times 60^{\circ}$ and $(2 n+1) \times 60^{\circ}$ (in all cases $0 \leq n \leq 5$ ) (Table 1). Most of the polytypes reported to date belong to subfamily A. In subfamily B, only $2 \mathrm{M}_{2}$ and $2 \mathrm{O}$ are known, the latter being rare. A few examples of mixed-rotation polytypes have been reported: $4 A_{1}$ (Takeda, 1967), $3 M_{2}$ and $6 M_{1}$ (Bailey and Christie, 1978; Rule et al., 1987), 36 $A_{1}$ (Kogure and Nespolo, 1999a), and a disordered mixed-rotation sequence (Kogure and Nespolo, 1999b). As shown by Ross et al. (1966), a portion of the stacking sequence of the inhomogeneous mica polytypes coincides with one or more periods of one of 


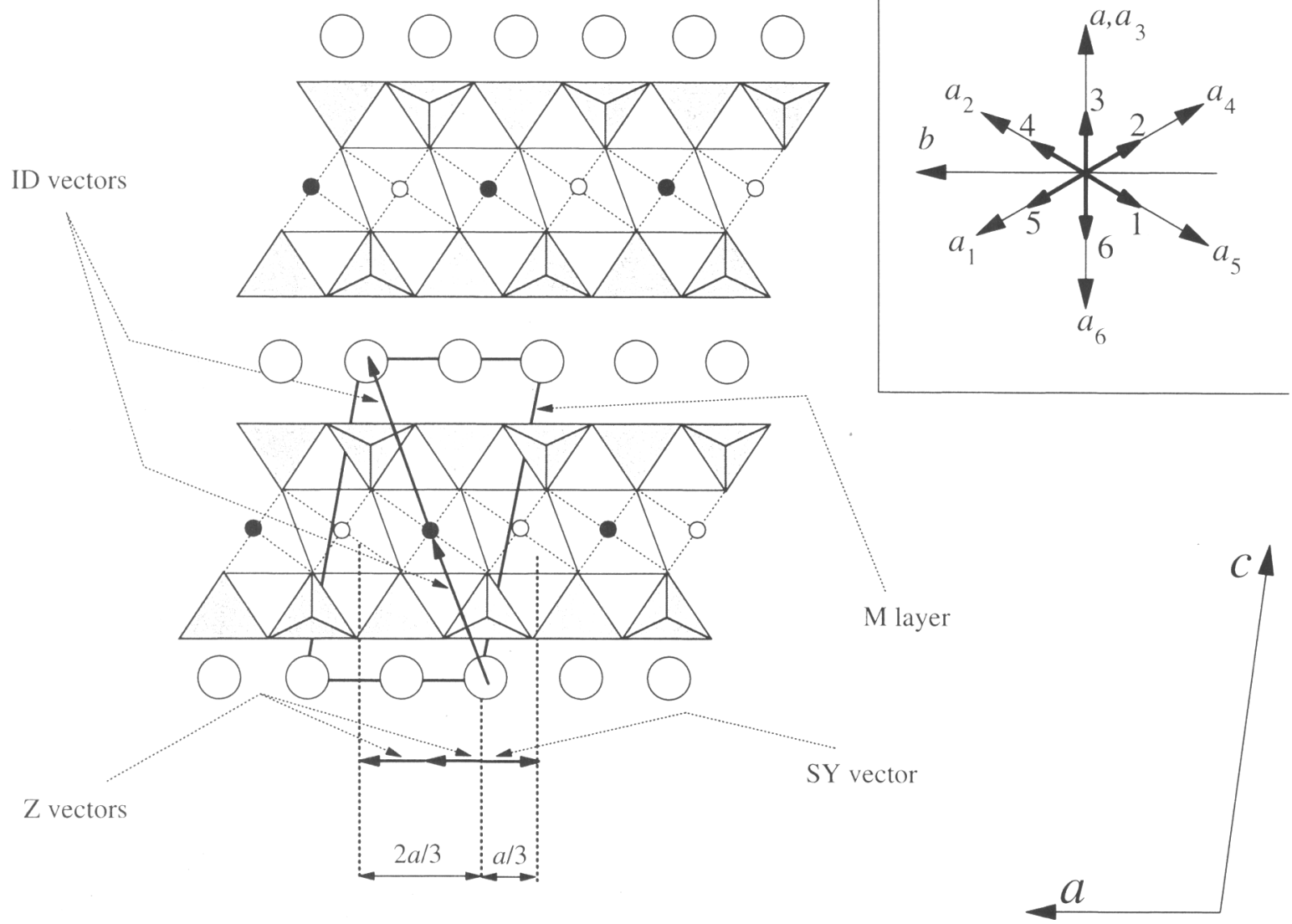

Figure 1. The structure of $1 M$ mica projected along the $b$ axis. Closed and open small circles represent octahedral sites $M 1$ and $M 2$, respectively. Large open circles are interlayer cations. The period along $c$ is compressed. Inset in the top-right corner: axes $(a, b)$ of the space-fixed reference $\left(C_{1}\right.$ setting) and of the structure-related references in the six possible orientations $\left(a_{1}-\right.$ $a_{6}$ ), and corresponding $Z$ symbols. The direction of the intralayer displacement (ID) vector and of its (001) projection ( $Z$ vector) is indicated by $Z$ symbol $\mathrm{i}(\mathrm{i}=1-6)$ when the $a_{\mathrm{i}}$ axis of the corresponding half-layer is parallel to the space fixed $a$ axis (modified after Nespolo et al., 1999a).

the three homogeneous subfamily-A polytypes, namely $1 M, 2 M_{1}$, and $3 T$, similarly to what occurs in $\mathrm{SiC}$ polytypes. For this reason, these three polytypes are called basic structures (Baronnet and Kang, 1989). The remaining portion of the stacking sequence of an inhomogeneous polytype represents a deviation in the stacking sequence of the basic polytypes. Inhomogeneous polytypes are thus said to belong to one of the three structural series represented by the basic structures (Ross et al., 1966; Takeda and Ross, 1995).

The purpose of the present paper is to introduce a model to explain in a simple way, within the framework of the spiral growth mechanism of Frank (1949, 1951a), the appearance of complex stacking sequences that cannot be accounted for by previous models. The key aspect of this model is the distinction between two kinds of layers, M1 and M2, which differ for the cation distribution in the $O$ sheet. Each layer can be transformed into the other through a growth defect or a crystallographic slip at the octahedral sheet. A brief summary of the present knowledge is necessary to introduce the new model.

\section{STRUCTURAL CONTROL}

Smith and Yoder (1956) introduced the concept of structural control to correlate the observed frequency of mica polytypes with chemical composition. Basic considerations for structural control are summarized as follows (Guiven, 1971; Baronnet, 1980). 1) In the absence of structural control, the frequency of occurrence of a polytype with a given number $N$ of layers should be a simple function of the number of layers in the repeat unit. This hypothesis contrasts with experimental observations. 2) Structural control is related to crystal chemistry and to the geometrical distortions of the layer, especially of the $O$ sheet. Distortions limit the stacking possibilities and, therefore, the formation of a great number of polytypes. Distortions are stronger in pure dioctahedral micas, lower in Li-rich trioctahedral micas, and still lower in Li-poor triocta- 


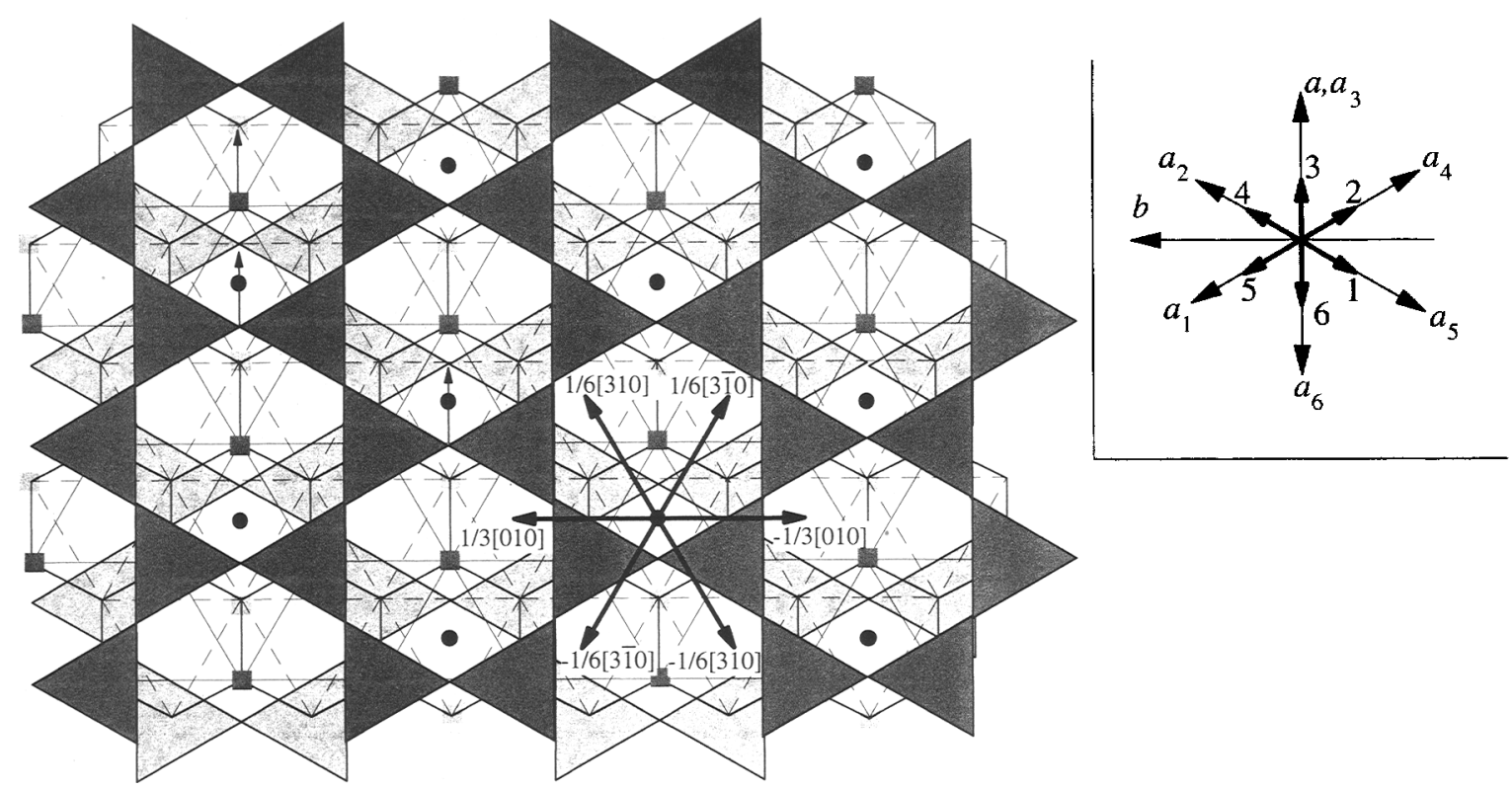

Figure 2. Projection of the M layer onto the (001) plane. The $T$ sheets are drawn in their ideal symmetry $P(6) m m$, i.e., for zero ditrigonal rotation. Squares represent interlayer cations and $(\mathrm{OH}, \mathrm{F})$ groups [superposed in the (001) projection]. Dark and light gray indicates positions above and below the $O$ sheet, respectively. $M 1$ sites are shown by black circles. The six vectors connecting $M 1$ sites with the neighboring $M 2$ sites have length $|b| / 3$ (see text).

hedral micas. The number of inhomogeneous polytypes is consistent with the above sequence 3 ) Structural control in trioctahedral micas should be not sufficiently strong to prevent the modification of the original stacking sequence, but it should be sufficiently strong to maintain "memory", during the growth of the crystal, of the stacking sequence of the matrix.

The causes of this stacking memory in the basic structures are not well understood. Structural control may be related to the details of the $\mathrm{M}$ layer, according to desymmetrization (Ďurovic, 1979): the symmetry of a layer with given composition depends on its stacking in a polytypic structure and is commonly lower than in the single-layer structure. Energy differences owing to polytypic details are by definition small. However, when all the other factors are equivalent, these differences may be sufficient to control the original stacking sequence.

Whatever the reasons for structural control, both the high frequency of occurrence of the three basic structures and the presence of their stacking sequences as sub-periods in those of the inhomogeneous polytypes favor a mechanism where the inhomogeneous polytypes are formed through a modification of the basic structures.

\section{EFFECT OF THE CATION DISTRIBUTION IN THE $O$ SHEET: M1 VS. M2 LAYER}

In the $O$ sheet, there are three translationally independent cation sites: the $M 1$ site is trans-coordinated by $\mathrm{OH} / \mathrm{F}$ : where the layer is centrosymmetric, the cen- ter of symmetry is at the $M 1$ site. The remaining two sites are labeled $M 2$ and are cis-coordinated by $\mathrm{OH} / \mathrm{F}$; where the layer contains a symmetry plane, this plane relates the two $M 2$ sites (Figure 3). Where the symmetry plane is absent, the cis sites are subdivided into $M 2$ and M3 (Bailey, 1984). Based on the distribution of the cations in the $O$ sheet, the symmetry of the octahedral sheet may be $H(\overline{3}) 12 / m$ (homo-octahedral with the three sites occupied by the same average cation), $P(\overline{3}) 12 / m$ (meso-octahedral with one site occupied by an average cation different from that in the other two sites), or $P(3) 12$ (hetero-octahedral with all three sites occupied differently). The two $T$ sheets of a layer are staggered by $|a| / 3$ in (001) projection and the ideal layer symmetry of the $\mathrm{M}$ layer is $C 12 / m(1)$ (Dornberger-Schiff et al., 1982).

In meso-octahedral micas, the two average cations in the $O$ sheet can be distributed in three different ways in the three translationally independent cation sites. Similarly, in hetero-octahedral micas the three average cations can be distributed in six different ways. To distinguish layers with different cation distributions, the origin of the $O$ sheet is conventionally taken at the site with the unique cation (meso-octahedral micas) or at the site containing the average cation with the lowest electron density (hetero-octahedral micas). In most cases, the origin defined in this way corresponds to the $M 1$ site, but examples are also known of micas for which the origin of the $O$ sheet is either in the $M 2$ or in the $M 3$ site (Ďuroviž et al, 1984). Correspondingly, two types of layers, "M1" 
Table 1. Classification of mica polytypes in terms of the relative rotations between layers, and corresponding parity rules for $\mathrm{Z}$ and RTW symbols.

\begin{tabular}{|c|c|c|c|}
\hline Z symbols & RTW symbols & Relative rotations between layers & Kind of polytype \\
\hline $\begin{array}{l}\text { Same parity } \\
\text { (either all odd or all even) }\end{array}$ & all-even & $\begin{array}{l}0^{\circ}(1 M) \\
120^{\circ} \text { and } 240^{\circ}\left(2 M_{1}\right) \\
120^{\circ} \text { or } 240^{\circ}(37) \\
2 n \times 60^{\circ}, \text { with more than } \\
\quad \text { one value of } n\end{array}$ & $\begin{array}{l}\text { homogeneous subfamily A } \\
\text { (basic structures) } \\
\text { inhomogeneous subfamily A }\end{array}$ \\
\hline Alternating parity & all-odd & $\begin{array}{l}180^{\circ}(2 \mathrm{O}) \\
60^{\circ} \text { and } 300^{\circ}\left(2 \mathrm{M}_{2}\right)^{1} \\
60^{\circ} \text { or } 300^{\circ}(6 \mathrm{H}) \\
(2 n+1) \times 60^{\circ}, \text { with more than }\end{array}$ & homogenous subfamily B \\
\hline $\begin{array}{l}\text { Mixed, non-alternating } \\
\text { parity }\end{array}$ & both odd and even & $\begin{array}{l}\text { one value of } n \\
\text { both } 2 n \times 60^{\circ} \text { and } 2 n \times 60^{\circ}\end{array}$ & $\begin{array}{l}\text { inhomogeneous subfamily } \mathbf{B} \\
\text { mixed rotation } \\
\text { (inhomogeneous) }\end{array}$ \\
\hline
\end{tabular}

' Baronnet and Kang (1989) included also $2 M_{2}$ in their definition of basic structures, foreseeing the possible occurrence of inhomogeneous polytypes based on the stacking sequence of this polytype and belonging thus to the $2 M_{2}$ structural series. To date no example has been reported.

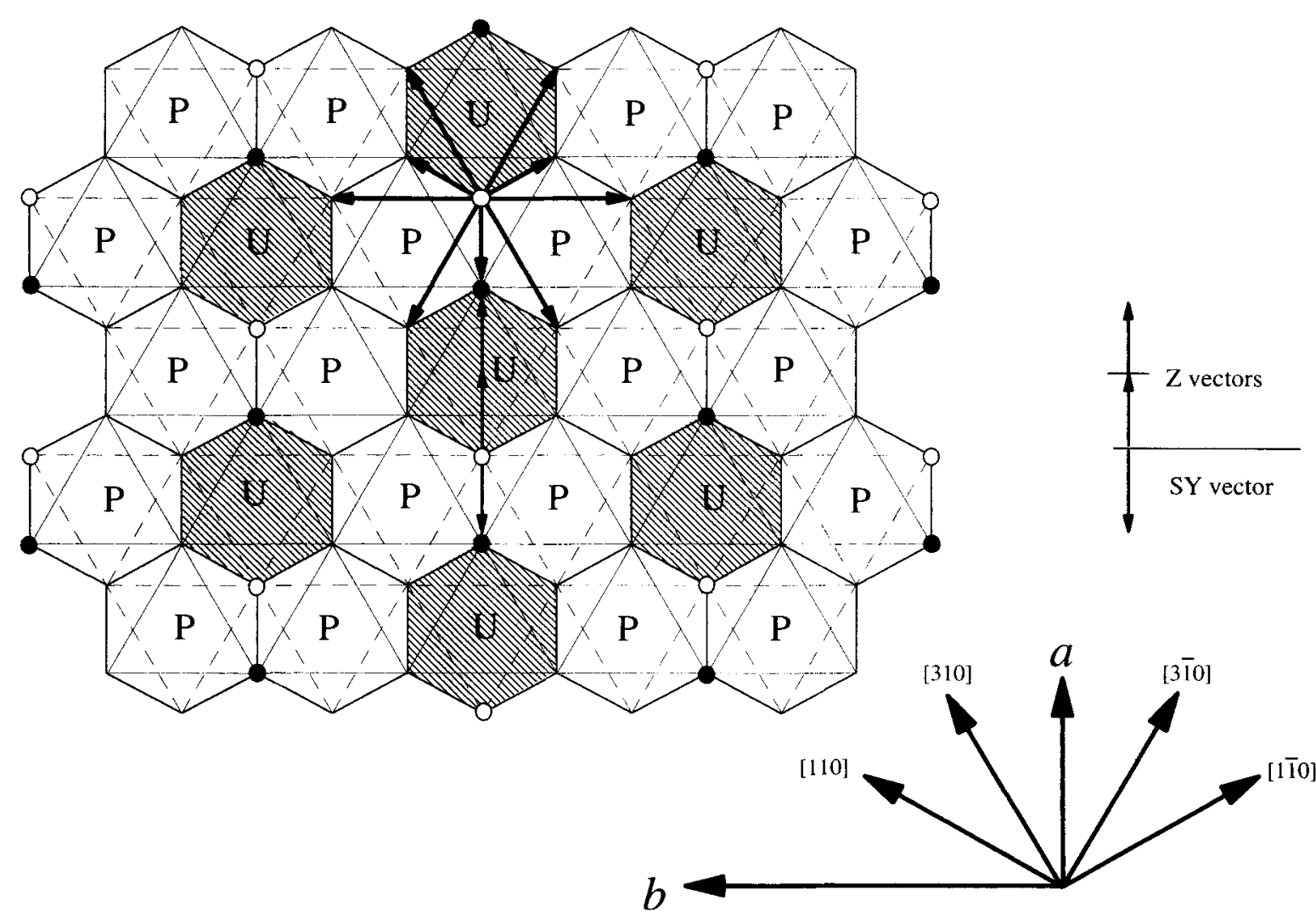

Figure 3. The octahedral sheet of a meso-octahedral mica seen in projection onto the (001) plane. Shaded octahedra are $M 1$ sites, with $(\mathrm{OH}, \mathrm{F})$ groups in trans configuration (open and close circles are OH,F groups below and above the cations, respectively). The $M 1$ site contains the unique cation (U), whereas the two $M 2$ sites contain a pair (P) of identical cations. The $Z$ vectors and the SY vector (vector sum of $Z$ vectors) are indicated by thin arrows (repeated on the right, for clearness). Thick arrows indicate the vectors of a crystallographic slip of the first sort (CSt: long vectors) and of the second sort (CS ${ }^{\mathrm{II}}$ : short vectors) at the lower plane. 


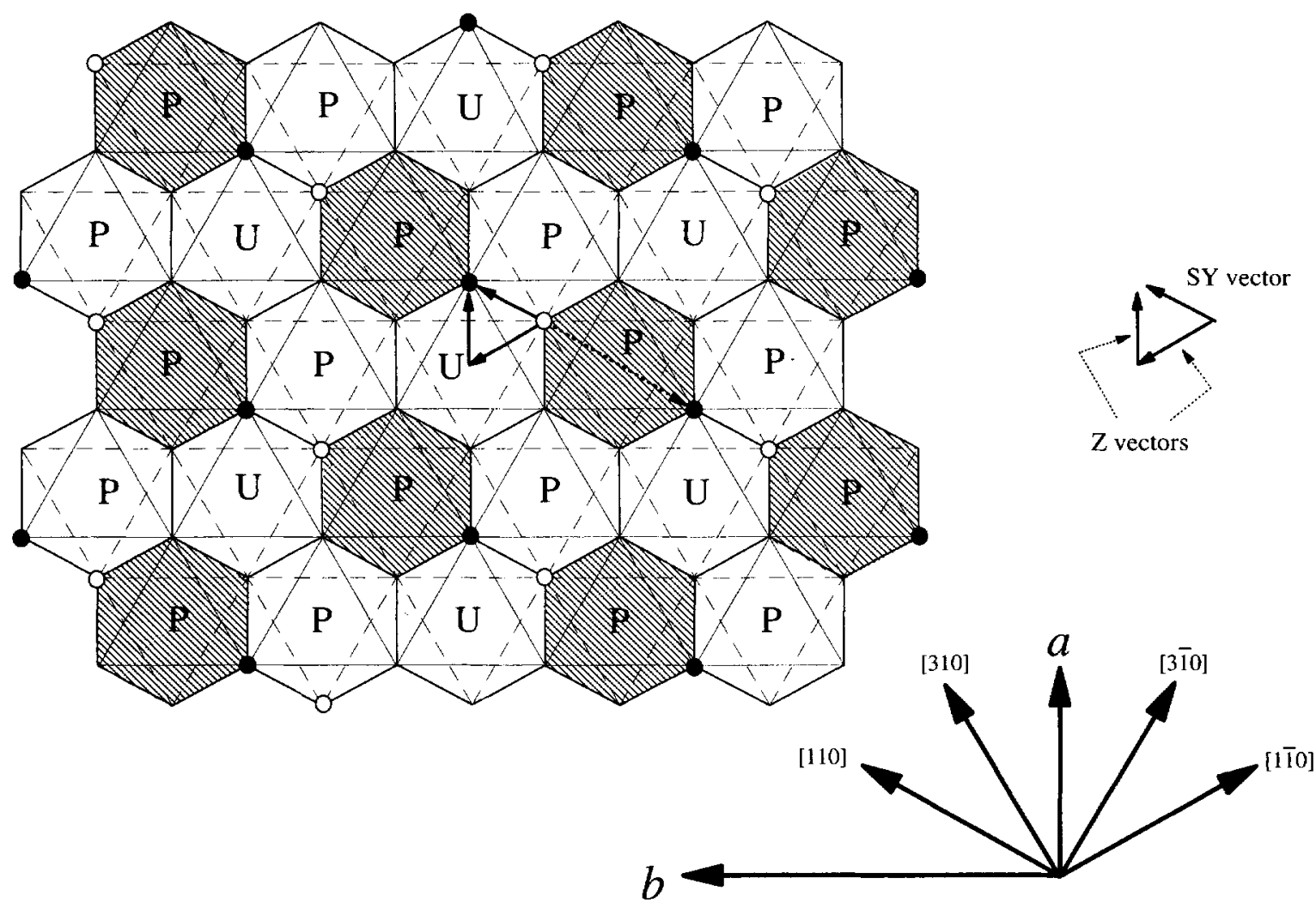

Figure 4. The (001) projection of an octahedral sheet of a meso-octahedral mica, after a $|b| / 3$ crystallographic slip of the lower oxygen-atom plane along any of [010], [310], and [310] directions. One of the two $M 2$ (unshaded) sites contains now the unique cation (U). The SY vector is rotated clockwise by $120^{\circ}$ with respect to that in Figure 3 . The vector translationaliy equivalent to SY (dashed), with length $2|a| / 3$, is also rotated clockwise by $120^{\circ}$ with respect to the pair of $\mathrm{Z}$ vectors in Figure 3 . SY vectors describe the M1 $\rightarrow$ M2 layer transformation as a simple rotation of the stacking vector and are not suitable to show the layer transformation.

and "M2", are distinguished (Nespolo et al., 1999a) (Figures 3 and 4). In homo-octahedral micas, the layer is always M1. For M1 and M2 layers, the ideal (highest) layer symmetry is $C 12 / m(1)$ and $C 12(1)$ respectively (Dornberger-Schiff et al., 1982; Zvyagin, 1997). These two kinds of layers can be distinguished only when the cation distribution is known, i.e., after completion of a structure refinement.

\section{VECTORS AND SYMBOLS TO DESCRIBE MICA POLYTYPES}

The stacking vector introduced by Smith and Yoder (1956) (hereafter termed "SY vector") is the (001) projection of the shortest vector connecting two interlayer cations located at the opposite sides of an $M$ layer (Figure 1). This vector is not related to the occupancy of the octahedral cation sites, and thus cannot be used to distinguish between $\mathrm{M} 1$ and $\mathrm{M} 2$ layers. The vectors introduced by Zvyagin et al. (1979) (hereafter termed " $Z$ vectors") are the (001) projections of pairs of intralayer displacement (ID) vectors (Figure 1). The latter connect the origin of the $O$ sheet with the nearest interlayer site and vice versa (the sequence of layers must be observed from the same direction). $\mathrm{Z}$ vectors can thus distinguish between M1 and M2 layers; they are defined in terms of a space-fixed, orthogonal reference, based on a pair of orthohexagonal axes $(a, b)$ in the plane of the layer $\left(C_{1}\right.$ setting in Nespolo et al., 1998) (Figure 2). The six possible orientations of the $\mathrm{Z}$ vectors are indicated by six structure-related $a_{\mathrm{i}}$ axes $(i=1-6)$. When the absolute orientation of a halflayer is such that its $a_{i}$ axis is parallel to the spacefixed axis $a$, the (001) projection of the corresponding ID vector is denoted by the $Z$ symbol $i$ ( $i=1,2, \ldots$ 6) (Figure 2). The complete $Z$ symbol represents the sequence of $Z$ vectors, and is expressed as $\mathrm{ij}_{0} \mathrm{kl}_{0} \mathrm{mn}$ $\ldots$, where 0 indicates the interlayer displacement vector, which in micas has components $(0,0)$ (the interlayer cations fix the relative positions of two successive $M$ layers). Pairs of $Z$ symbols give the orientation of the two halves of an $M$ layer ( $i$ and $j, k$ and $1, \mathrm{~m}$ and $\mathrm{n}, \mathrm{etc}$.): they must have the same parity (either even of odd), to produce an octahedral coordination for the cations. Micas comprised of M1 layers have 
two identical $Z$ symbols, i.e., $\mathrm{i}=\mathrm{j}, \mathrm{k}=\mathrm{l}, \mathrm{m}=\mathrm{n}$, etc.; in this case the symbol 0 may be omitted and a shortened symbol IKM ... is commonly adopted (Zhukhlistov et al., 1990; see Figure 1 therein). Adoption of the shortened $Z$ symbolism is equivalent to describe the layer as if it is homo-octahedral, and for this reason the term homo-octahedral approximation was introduced (Nespolo et al., 1999a).

Other symbols often used to describe mica polytypes are the orientation-free, rotational RTW symbols (Ross et al., 1966), which express the relative rotations $\left(\bmod 60^{\circ}\right)$ between pairs of SY vectors and cannot distinguish between M1 and M2 layers. The symbol correspondence for the three kinds of polytypes described in the previous section is given in Table 1. Hereafter, the indices of axes and planes are given in the (pseudo)orthohexagonal cell. This cell coincides with the conventional cell for orthogonal polytypes, and contains three conventional cells for non-orthogonal polytypes. Consequently, planes almost normal to $(001)$ are indicated as $(h k 0)$.

\section{SPIRAL GROWTH IN MICAS}

Hartman and Perdok (1955) classified crystal faces into $F$ (flat), $S$ (stepped), and $K$ (kinked) depending on whether they contain in the same order two or more, one, or no periodic bond chain (PBC) vectors, i.e., vectors indicating directions of strong bonding. In micas, the $\mathrm{K}$ faces (e.g., $\{111\}$ in the orthohexagonal setting) are not developed as crystal faces; the $F$ faces correspond to the $(001)$ plane, and the $S$ faces are normal (e.g., $\{100\})$ or inclined (e.g., $\{110\})$ to the $(001)$ plane (see, e.g., Peacock and Ferguson, 1943). The F faces grow slowly in the direction perpendicular to the face, are large and flat, have a low surface energy, and no dangling bonds, they often contain growth-spirals. In contrast, $\mathrm{S}$ faces grow rapidly in the direction perpendicular to the face, are relatively small, have a high surface energy, contain dangling bonds, and are the preferential site for the accretion of larger crystallization units, e.g., already existing crystal nuclei. S faces are characterized by striations and a lack of growth spirals (Sunagawa and Bennema, 1982). Cleavage in micas occurs along $F$ faces, the structure of $F$ faces is comprised of basal oxygen atoms and interlayer cations (Kogure, 1997).

The spiral-growth model is a perfect memory mechanism repeating either the complete stacking sequence of a polytype (perfect dislocation: the pitch of the screw dislocation from which the spiral originates is a multiple of the polytype periodicity) or a part of a stacking sequence (imperfect dislocation: the pitch of the screw dislocation is a submultiple of the polytype periodicity). The presence of a stacking fault in the exposed ledge increases the number of polytypes which can form in this way (Frank, 1949, 1951a). The outcrop of a screw dislocation on the surface of a crys- tal is the result of an increase in the crystal stress either because of thermal stresses or physical-type stresses, i.e., non-uniform distribution of impurities (Frank, 1951a).

Polytypism in binary compounds may be generally explained by considering the presence of one or more stacking faults in the exposed ledge (faulted matrix model, FMM; spiral growth of an unfaulted ledge: perfect matrix model, PMM) (for details see Baronnet, 1997). For micas, the PMM can explain only subfamily-A inhomogeneous polytypes belonging to $2 M_{1}$ and $3 T$ structural series, and the possible sequences correspond to RTW symbols $(22)_{n} 0\left(2 M_{1}\right.$ structural series), and $(222)_{\mathrm{n}} 0$ and $(222)_{\mathrm{n}} 22(3 T$ structural series) (Ross et al., 1966; Baronnet, 1980). The FMM introduces a higher degree of flexibility, without however explaining the more complex stacking sequence, which would require "singly- or multiply-faulted exposed ledges containing higher energy fault configurations" (Pandey et al., 1982), a situation with a low probability of occurrence and at variance with the starting assumptions of the FMM itself. Micas have features markedly different from those of binary compounds: 1) they are not based on closest-packed arrangements: only the planes of hydroxyl groups and apical oxygen atoms follow the closest-packed topology (Pauling, 1930), and micas have a large anionanion spacing in the plane of hydroxyl groups and apical oxygen atoms (Noe and Veblen, 1999); 2) layers are related by both translations and rotations; 3 ) basic structures include one, two, and three-layer polytypes only; and 4) the relative positions of successive layers are determined by the interlayer cations. Other factors, different from the casual presence of stacking faults in the ledge, must be considered if Frank's mechanism of spiral growth is to explain the most complex stacking sequences reported in micas.

\section{RELATION BETWEEN CRYSTALLIZATION ENVIRONMENT, CRYSTAL CHEMISTRY, AND THE OCCURRENCE OF INHOMOGENEOUS POLYTYPES}

The explanation for the occurrence of long-period polytypes with a complex stacking sequence is related to a perturbation occurring during spiral growth. Wellknown perturbing events include the interactions between spirals present on the same crystal, between a spiral and a crystal, or between two separate crystals. The relation between these three types of interactions and the frequency of inhomogeneous polytypes can be explained in terms of crystallization environments.

During metamorphism, minerals are formed in situ by solid-solid transformation and the products occupy the space of the reactants. Spiral growth is commonly not observed on crystal surfaces (Sunagawa et al., 1975). Dioctahedral micas are typical of metamorphic rocks (Guidotti and Sassi, 1998) and thus far they are 
not known to form inhomogeneous polytypes [the three-layer monoclinic muscovite of Axelrod and Grimaldi (1949) is a $3 T$ polytype (Smith and Yoder, 1956)]. Also, in synthetic samples, the occurrence of inhomogeneous polytypes in dioctahedral micas is exceptional (Baronnet et al., 1976). Li-rich trioctahedral micas are typical of greisens, pegmatites, and hydrothermal veins (Rieder et al., 1970) and are thus formed by metasomatism, through dissolution-precipitation. In these environments, crystals precipitate directly from slow-moving solutions with low supersaturation. Polygonal growth spirals are invariably present on their surface (Sunagawa, 1977). Some inhomogeneous polytypes belonging to $1 M$ or $3 T$ structural series were reported, and the stacking sequences were closely related to those of the corresponding basic structures (Takeda, 1967; Rieder, 1970). Li-poor trioctahedral micas occur mainly in magmatic environments, and inhomogeneous polytypes were reported in both plutonic (Borutskiy et al. 1987; Zhukhlistov et al., 1990, 1993; Bigi and Brigatti, 1994) and volcanic rocks (Hendricks and Jefferson, 1939; Amelinckx and Dekeyser, 1953; Ross et al., 1966; Takeda and Ross, 1995; Nespolo and Takeda, 1999; Kogure and Nespolo, 1999a).

In plutonic Li-poor trioctahedral micas, the reported polytypes, even when they possess a long-period stacking sequence, are a simple modification of one of the three basic structures. Inhomogeneous polytypes with more complex stacking sequences are found in druses or in volcanic fumaroles, where crystallization occurs by post-volcanic action (e.g., Tomisaka, 1958, 1962; Ross et al., 1966): their occurrence seems thus related to the final differentiation stage of a magma, where volatile components are present as super-critical vapor phase. Experiments simulating the growth in these environments have successfully produced inhomogeneous polytypes of fluor-phlogopite, emphasizing the role kinetics play in crystal growth (Sunagawa et al., 1968).

The main features of crystal growth in a magma and from a vapor phase can be summarized and compared as follows (Sunagawa, 1977, 1978, 1982; Sunagawa and Bennema, 1982). 1) Solid-fluid interactions are stronger during the crystallization from a melt then during precipitation from a vapor phase, for which accretion of groups of atoms or ions instead of single atoms or ions is characteristic. 2) The critical supersaturation below which the spiral growth mechanism is active is much higher with growth from the vapor phase than from solutions. Growth spirals are thus more numerous and frequent in vapor growth, and much less developed in crystallization from magma. 3) The mobility of crystal nuclei and the probability to settle on larger crystals is much higher in a fluid or vapor phase than in a silicate magma. In a vapor phase, small mica crystals move freely in the open space, like "flying magic carpets" (Sunagawa and Endo, 1971; Sunagawa and Tomura, 1976).

Free moving crystal nuclei in a fluid environment frequently come into contact with each other and occasionally get attached. Just before agglutinating, they adjust their mutual orientation, to form a parallel growth, a twin/allotwin or, more rarely, a plesiotwin (Sunagawa et al., 1975; Sunagawa and Tomura, 1976) (Allotwins are the twin-like oriented crystal associations of different polytypes of the same compound; see Nespolo et al., 1999b; plesiotwins are oriented crystal associations based on a large coincidence-site lattice; see Nespolo et al., 1999c). Small mica nuclei settle onto the $F$ face of a larger mica crystal, taking a crystallographically identical, twinned, or inclined orientation relative to the host crystal, with composition plane (001), as observed in the "biotitic phlogopite" (now termed "ferrous phlogopite", Rieder et al., 1998) from druses at Mutsure-jima, Japan (Sadanaga and Takéuchi, 1961; Sunagawa, 1964; Sunagawa and Tomura, 1976). This specimen (Kôzu and Yoshiki, 1929; Kôzu and Tsurumi, 1931; Tomisaka, 1958) was the object of a systematic surface study by phase-contrast microscopy and was described as a $1 M$ polytype (Tomisaka, 1962), but no systematic diffraction or high-resolution transmission electron microscopy (HRTEM) study was performed, and thus the sample may contain other polytypes. If the crystallites settle close to each other, they may interact through their $S$ faces. This kind of contact eliminates two high-energy surfaces with exposed dangling bonds (one for each crystal) to form a twin with a composition plane (nearly) normal to the (001) plane (see examples in Sunagawa, 1964). The surface of the larger crystal where they settle may not be atomically flat and the crystallites will in general cover an irregularly stepped surface, thus producing an inclined interface. Two crystallites close to each other, in general, are thus relatively inclined. One or more screw dislocations will, therefore, arise along the boundary of two inclined crystallites which coalesce along their $S$ faces, from which new growth ledges originate (Sunagawa et al., 1975; Tomura et al., 1979). A cooperative growth begins which, during its early stage, must overcome a certain degree of mismatch caused by the relative inclination of the crystallites. As discussed below, it is through the recovery of this mismatch that an inhomogeneous polytype with a relatively complex stacking sequence can form.

\section{POLYTYPE FORMATION AND MECHANICAL STRENGTH: CRYSTALLOGRAPHIC SLIPS AND THEIR ROLE IN THE M1 $\rightarrow$ M2 TRANSFORMATION}

The $\mathrm{M}$ layer may form initially as an M2 layer: for dioctahedral micas, this occurs when the grain crystallizes in conditions far from equilibrium (Zvyagin et 


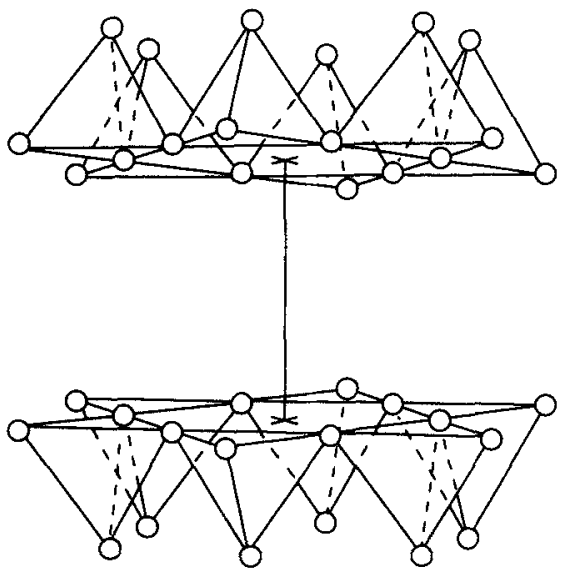

(a)

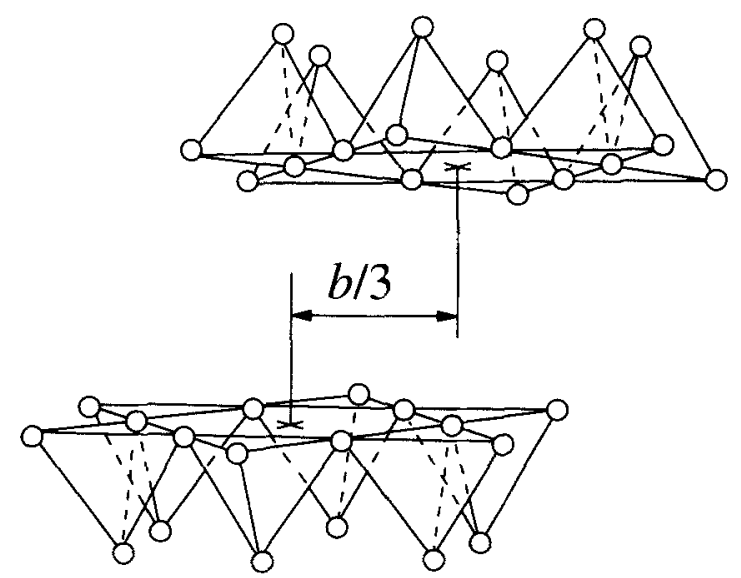

(b)

Figure 5. The trigonal (ideally hexagonal) rings of two tetrahedral sheets belonging to adjacent layers where interlayer cations occur. In (a) the two rings match and the cation occurs. In (b) there is a $|b| / 3$ mismatch between the two rings. Two successive $M$ layers where trigonal rings of the adjacent $T$ sheets do not superpose in (001) projection make a shifted pair. The occurrence of an interlayer cation between a shifted pair is an unfavorable coordination because the cation occurs near the edges of tetrahedra of both adjacent layers.

al., 1985; Bloch et al., 1990; Zhukhlistov and Zvyagin, 1991; Zhukhlistov et al., 1996). However, the M2 layer can also be obtained by transformation from an M1 layer. Important information on the M1 $\rightarrow$ M2 layer transformation is obtained by considering the mechanical strength and the occurrence of slips in the micas.

Notwithstanding the wide-ranging literature on edge dislocations in micas which started with Silk and Barnes (1960), the location of slip planes was partly overlooked. Only basal slip, i.e., slip along the (001) plane, occurs in micas. Low-energy displacements correspond to slip vectors of $\pm[100], \pm 1 / 2[110]$, and $\pm 1 / 2$ [110] (length $|a|$ ), which connect two interlayer cation sites (e.g., Caslavsky and Vedam, 1970; Etheridge et al., 1973; Baños et al., 1983; Kronenberg et al., 1990). Displacements with slip vectors $\pm[010], \pm 1 / 2[310]$, and $\pm 1 / 2[3 \overline{1} 0]$ (length $|b|$ ) require higher energy and are considered the result of multiple slip systems (Christoffersen and Kronenberg, 1993). Bell and Wilson $(1977,1981,1986)$, Wilson and Bell (1979), and Noe and Veblen (1999) demonstrated that slips occur also in the $O$ sheet, with slip vectors of $\pm 1 / 3[010], \pm 1 / 6$ [310], and $\pm 1 / 6[3 \overline{1} 0]$ (length $|b| / 3$ ), which connect an $M 1$ site with one of the nearest $M 2$ sites (Figure 2). These slips preserve the octahedral coordination of the cations but modify the (OH,F) positions in one of such planes within a layer. $A$ slip with a fault vector length of $|b| / 3$ in the interlayer region is highly improbable, because it would create a highly unstable coordination environment for the interlayer cations (Figure 5).

The biotite studied by Bell and Wilson (1977, 1981, 1986) and Wilson and Bell (1979) was more susceptible to deformation than coexisting muscovite, but contained a low concentration of $F$. In contrast, the biotite investigated by Kronenberg et al. (1990) and Mares and Kronenberg (1993) was stronger than muscovite, but was F-rich. Dahl and Dorais (1996) have interpreted this apparent contradiction on the basis of the different content of fluorine. In trioctahedral micas, the $\mathrm{OH}$ dipole is perpendicular to the (001) plane, directed towards the interlayer cation (Bassett, 1960). The $\mathrm{OH} \rightarrow \mathrm{F}$ substitution at the $\mathrm{Mg}$ sites removes the $\mathrm{OH} \cdots \mathrm{K}$ repulsion, strengthens the $\mathrm{K}-\mathrm{O}$ bonds, and explains the unusual mechanical strength of fluorine-rich biotites (Van Valkenburg and Pike, 1952; Takeda and Donnay, 1966; Takeda et al., 1971). A similar but more complex phenomenon occurs in oxybiotite, where strengthening of the $\mathrm{K}-\mathrm{O}$ bonds comes from the $\mathrm{OH} \rightarrow \mathrm{O}$ substitution (Barshad and Kishk, 1968; Farmer and Wilson, 1970) related to the $\mathrm{Fe}^{2+} \rightarrow \mathrm{Fe}^{3+}$ oxidation and the $\left(\mathrm{Fe}^{2+}, \mathrm{Mg}^{2+}\right)_{-2} \mathrm{H}_{-2} \mathrm{Ti}_{2}$ exchange (Filippov et al., 1976). In addition, the $\mathrm{OH}$ dipoles surviving after the oxidation reaction to form oxybiotite deviate from the normal to (001) (Juo and White, 1969). Differently from the case of F-rich biotites, in oxybiotites, presence of high-charge cations $\left(\mathrm{Ti}^{4+}\right.$, $\mathrm{Fe}^{3+}$ ) destabilizes the octahedral sheet. As a conse- 


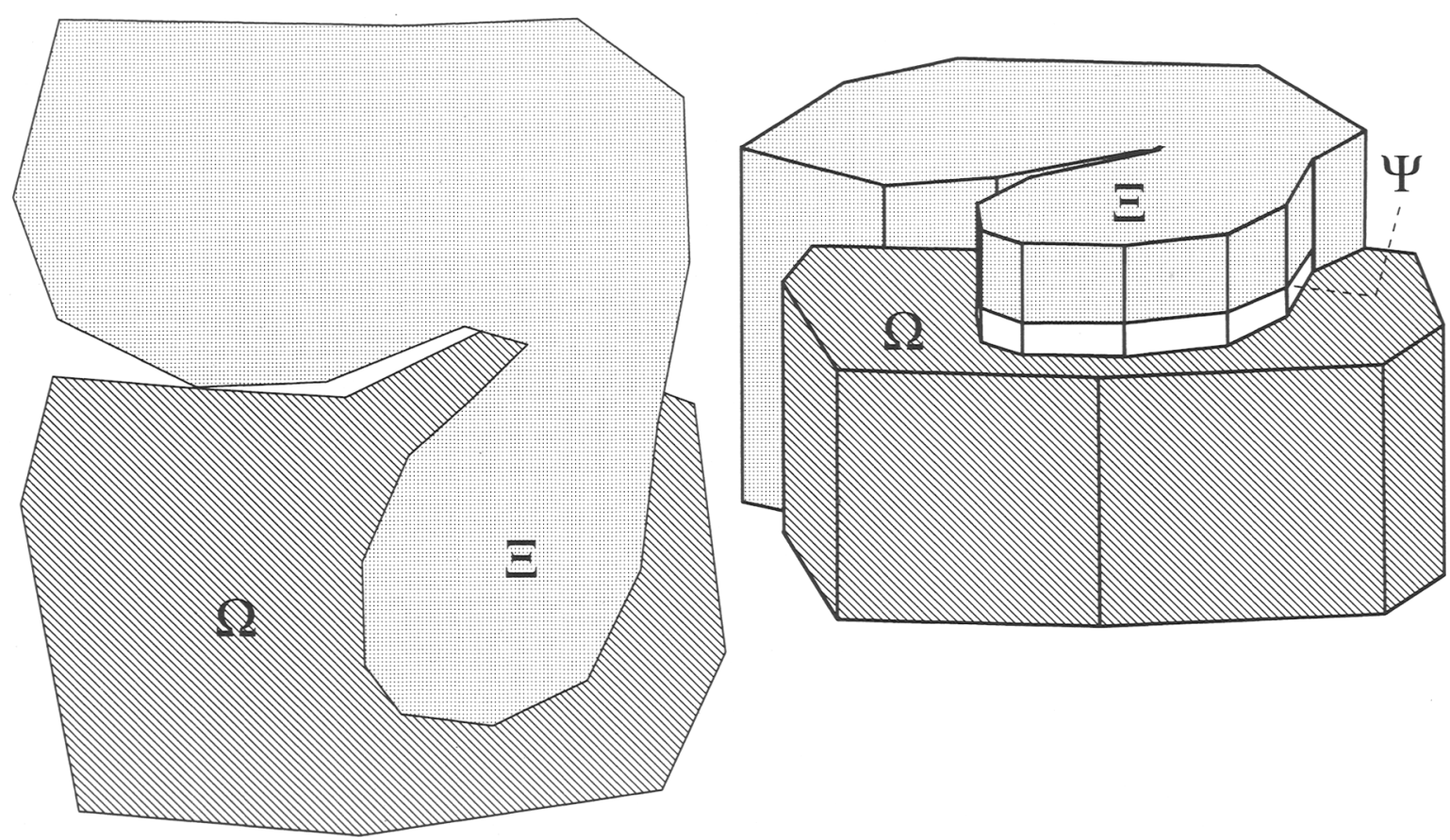

Figure 6. Simplified scheme of the perturbative theory of mica-polytype formation. Two components $\Omega$ and $\Xi$, here represented as two crystals attached laterally, interact and some growth layers from $\Xi$ spread onto the surface of $\Omega$. The lowest layer of $\Xi$ is modified because of a mismatch of the interlayer-cation positions between the two components, and the stacking vector takes an orientation different from the original vector $(\Psi)$. An inhomogeneous polytype results, whose stacking sequence is represented as $(\Omega)_{n} \Psi(\Xi)_{n}$, where $n$ layers and $n^{\prime}$ layers from $\Omega$ and $\Xi$, respectively, are connected by layer $\Psi$.

quence, the relative "hardness" of the interlayer and of the octahedral sheet is greatly modified and deformation occurs rather through a $|b| / 3$ slip in the $O$ sheet than through an $|a|$ slip in the interlayer.

The $|b| / 3$ slip in the $O$ sheet has an important structural consequence: it transforms an M1 layer into an M2 layer (Figures 3 and 4) and changes the corresponding stacking vector. The involvement of slips in the polytypic transformation of micas was suggested by Takéuchi and Haga (1971), who distinguished two types of crystallographic slips (CS) in the $O$ sheet. As noted by Takeuchi (1997), the word slip is more suitable than the word shear, because the latter potentially implies lattice deformation, whereas lattice is invariant under the mechanism discussed here. The commonly used abbreviation CS (Chisholm, 1975) should thus be read as crystallographic slip rather than crystallographic shear. The two types of CS are: 1) slip of the first sort ( $\left.\mathrm{CS}^{1}\right)$ : a $|b| / 3$ displacement of one of the planes of apical oxygen atoms with respect to other planes, maintained in a fixed position. The $\mathrm{CS}^{\mathrm{I}}$ of the apical oxygen atoms obviously implies a concomitant shift of the tetrahedra linked to them, in an M1 $\rightarrow$ M2 transformation of the $M$ layer. The two $Z$ vectors are rotated by $\pm 120^{\circ}$ (the $\mathrm{CS}^{\mathrm{I}}$ can occur in two opposite directions). Their vector sum coincides with the SY vector (length $|a| / 3$ ) and is translationally equivalent to a vector with length $2|a| / 3$, rotated $\pm 120^{\circ}$ from the original pair of $Z$ vectors (Figures 3 and 4). 2) Slip of the second sort $\left(\mathrm{CS}^{\mathrm{II}}\right)$ : this corresponds to a simultaneous displacement of $|a| / 3$ of both the planes of apical oxygen atoms in opposite directions, which reverses the slant of the octahedra. A displacement of only one apical oxygen plane would transform the octahedron into a trigonal prism and is, therefore, impossible.

In the present paper, the occurrence of $\mathrm{CS}^{\mathrm{I}}$ as a mechanism for polytype formation, instead of transformation, is proposed. The CSI may, in some cases, operate as a structural adjustment that is required when an external modifier influences the normal spiral growth of a basic polytype structure.

\section{PERTURBATION OF THE SPIRAL GROWTH THROUGH TWO-COMPONENT INTERACTION}

The stacking sequence of inhomogeneous mica polytypes can be interpreted as combinations of stacking sequences $\Omega$ and $\Xi$ of two basic structures. $\Psi$ is the stacking vector which connects the two sequences. The entire polytype can be represented by the symbol $(\Omega)_{n} \Psi(\Xi)_{n}$, where $n$ and $n$ ' are the number of layers in $\Omega$ and $\Xi . \Omega$ and $\Xi$ may originally be two interacting components (two spirals or crystals): the growth of $\Omega$ is modified by its interaction with $\Xi$ and possibly vice versa. $\Psi$ appears because of the eventual necessity of a structural adjustment at the boundary between the $\Omega$ and $\Xi$ (Figure 6). 

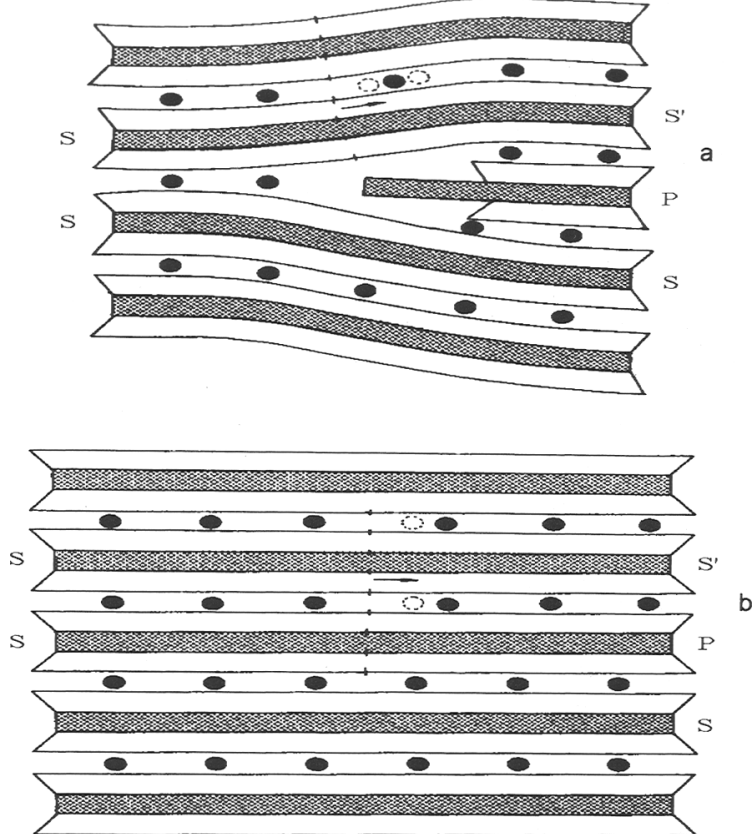

Figure 7. Recovery of the original stacking sequence of a crystal growing by spiral growth when disturbed by the deposition of a platelet (P) on the surface. (a) The spiral covers the platelet by elastic deformation; (b) some of the layers in the exposed ledge of the spiral are stopped by the deposited platelet; the remaining layers protrude on the surface of the platelet. In both cases, rearrangement of the protruding spiral may be necessary (shown by arrows) to match the interlayercation position. $S$ : original spiral; $P$ : platelet; $S^{\prime}$ : spiral as modified after intersecting the platelet (courtesy of $\mathrm{H}$. Takeda).

Three kinds of interactions are conceived: spiralspiral, spiral-crystal, and crystal-crystal.

\section{Spiral-spiral interaction}

The interaction of spirals is broadly classified into three general cases (Verma, 1953; Lemmlein and Dukova, 1956; Sunagawa and Bennema, 1982). 1) Two screw dislocations of the same sign with a separation larger than $19 \mathrm{r}^{*} / 2$, where $\mathrm{r}^{*}$ is the radius of the critical two-dimensional nucleus, produce independent spiral steps which, after a few turns, result in a system of parallel, nonintersecting steps. 2) Two screw dislocations of the same sign with a separation smaller than $19 \mathrm{r} * / 2$ produce a composite spiral in which an exchange of the spiral centers occurs at every turn. 3) Two screw dislocations of the opposite sign produce a closed loop and the spirals annihilate.

The most common mechanism by which composite spirals form is the decomposition of a single screw dislocation with a large Burgers vector into a cluster of screw dislocations with shorter Burgers vectors. The former is commonly created when impurities on the growing surface act as obstacles and the advance- ment of the growth layers is forced to stop, whereas other parts of the growing fronts continue to advance. As a result, impurities are entrapped by the growing layers, creating hollow dislocations (Frank, 1951b). For micas, hollow dislocations are observed if the Burgers vector exceeds a critical value of $20-40 \AA$ (Baronnet, 1972). The larger the Burgers vector, the less stable the dislocation, and the higher the probability that the dislocation decomposes into two or more smaller dislocations close to each other, which can form composite spirals. A composite spiral generated from the interaction of two or more spirals in parallel orientation and corresponding to the $1 M$ polytype does not produce a new stacking sequence. A new sequence can, however, be obtained when at least one of the spirals corresponds to a polytype with a zigzag stacking sequence (i.e., different from $1 M$ ). In this case, a spiral turn decomposes into two or more unit layers. The advancing rate differs with the advancing direction: it is faster along the stagger direction and slower normal to it. Consequently, interlacing is commonly observed (Frank, 1951a; Verma, 1953; Sunagawa and Koshino, 1975; Tomura et al., 1979). The different velocity along each direction creates a difference also in the relative position of the advancing front.

Another way to produce a modified stacking sequence is through the interaction of twinned spirals, i.e., growth spirals rotated at angles of $n \times 60^{\circ}$ on the same plateau, such as those observed on phlogopite crystals by Sunagawa and Tomura (1976). Where two twinned spirals, even those corresponding to a $1 M$ polytype, interact to form a composite spiral, the different orientation of the layers can produce a new stacking sequence, which depends on the number of layers from each spiral, on the relative orientation of the spirals, and on the advancing rate of the spiral steps.

\section{Spiral-crystal interaction. Platelet deposition}

Thin crystal nuclei deposited onto the surface of a larger crystal may begin growing cooperatively with a second component, which can be either an active spiral of the larger crystal on which it deposited, or another thin crystal deposited onto the same surface. The first case corresponds to the "platelet mechanism" introduced by Takeda and Ross (1995).

If the ledge of the growing spiral is higher than the platelet, the former covers the latter either via elastic deformation (Figure 7a) or by substitution of the lowest $N$ layers (where $N$ is the number of the layers in the platelet) of the spiral with the $N$ layers of the platelet (Figure 7b). The first mechanism is likely only for a very thin platelet (one or two layers thick). A deposited platelet can occur in one of six stable orientations with respect to the surface. If it occurs in a $(0$, $\pm 120^{\circ}$ ) orientation, the modified sequence corresponds to a subfamily-A polytype. In contrast, if the platelet occurs in $\left( \pm 60^{\circ}, 180^{\circ}\right)$ orientation, the modified se- 


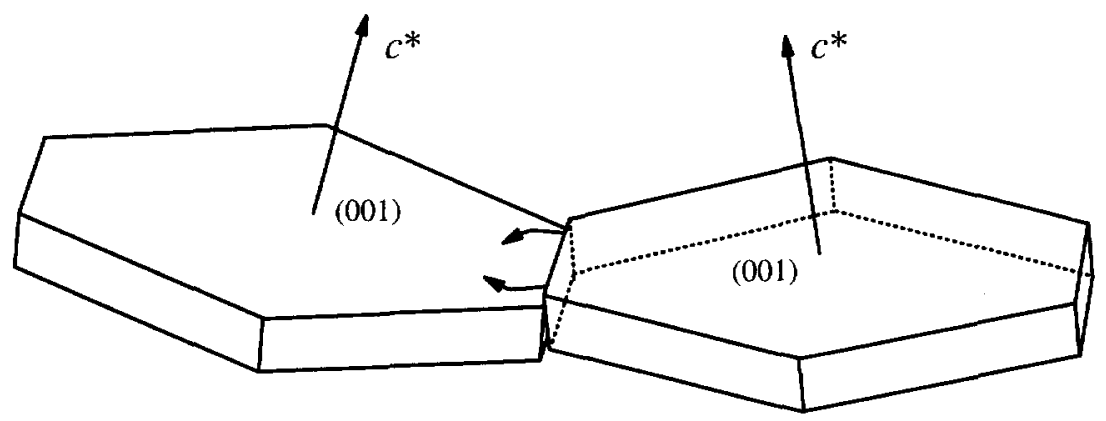

(a)
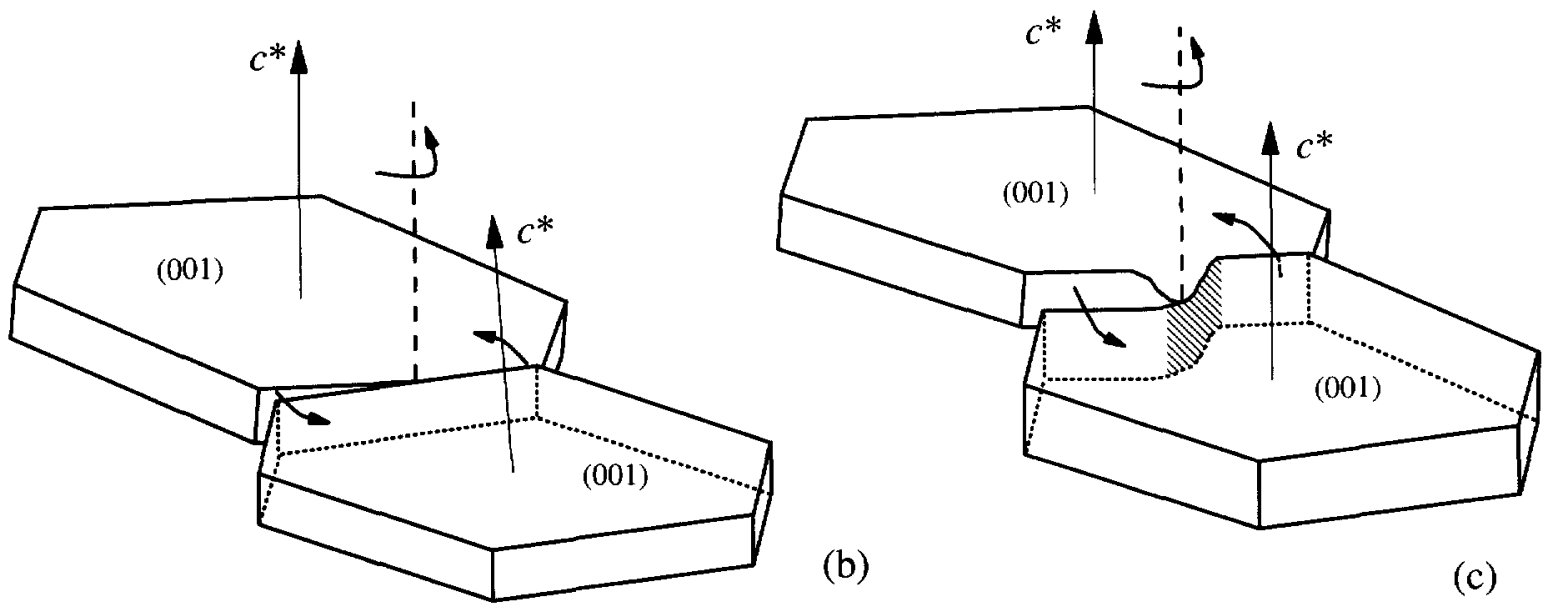

(b)

(c)

Figure 8. Imperfect lateral attachment of two crystallites, attached onto an $\mathrm{F}$ face of a larger crystal. The misorientation between the two crystals can be analyzed in terms of three limiting cases. (a) The two crystallites have their $c^{*}$ axes inclined towards each other. (b) The two crystallites have their $c^{*}$ axes in parallel planes but not equally oriented. (c) The two crystallites have their $c^{*}$ axes parallel but the plane of interface is kinked. At the contact point one or several layers can spread onto the surface of the adjacent crystallite. The presence of kinking or bending (c) concentrates the incoherence to a very narrow region; outside this region the interface between the two crystals may be coherent and several layers may protrude onto the surface of the neighbor crystal ( $c f$. ., Baronnet, 1973). Dashed lines indicate a screw dislocation formed by imperfect attachment of the crystallites ( $c f .$, Penn and Banfield, 1998).

quence corresponds to a mixed-rotation polytype. This is the most probable mechanism by which fluorophlogopite-4A $A_{5}(Z=6614, \mathrm{RTW}=0132)$ (Takeda, 1967) formed.

\section{Crystal-crystal interaction. Lateral coalescence of crystals and their cooperative growth}

In general, crystallites coalescing onto the same surface of a larger crystal are imperfectly attached, mainly because of the inclination with respect to the $F$ face of the crystal acting as substrate. Three limiting cases of imperfect attachment of two crystallites can be distinguished. 1) Their $c^{*}$ axes are not parallel but slightly inclined towards each other (Figure 8a). 2) Their $c^{*}$ axes are not parallel but lie within parallel planes (Figure 8 b). 3) Their $c^{*}$ axes remain parallel but one or both crystals are kinked (Figure 8c). The real case of imperfect attachment in most cases corresponds to a combination of these three cases. One or more dislo- cations with mainly a screw component, and Burgers vectors nearly parallel to [001], arise along the boundary. Consequently, layers from one crystal can crystallize onto the surface of the other crystal. Coherency requirements at the interface allow only very small misorientations and, as a consequence, only very few layers can protrude onto the surface of the neighbor crystal. In Figure $8 \mathrm{c}$, the kink permits a larger number of layers to spread. Mica crystals are frequently kinked or bent (Etheridge and Hobbs, 1974; Bell et al., 1986). These physical defects may produce an interface that is coherent, except in the small kinked or bent region.

Different type of interactions between coalescing crystallites can be distinguished. From a basic structure belonging to subfamily $\mathrm{A}$, either a subfamily-A or a mixed-rotation polytype can arise. A mixed-rotation polytype may be obtained also by the introduction of layers with $2 n \times 60^{\circ}$ (subfamily A) orientation into a subfamily-B homogeneous polytype. Instead, in- 


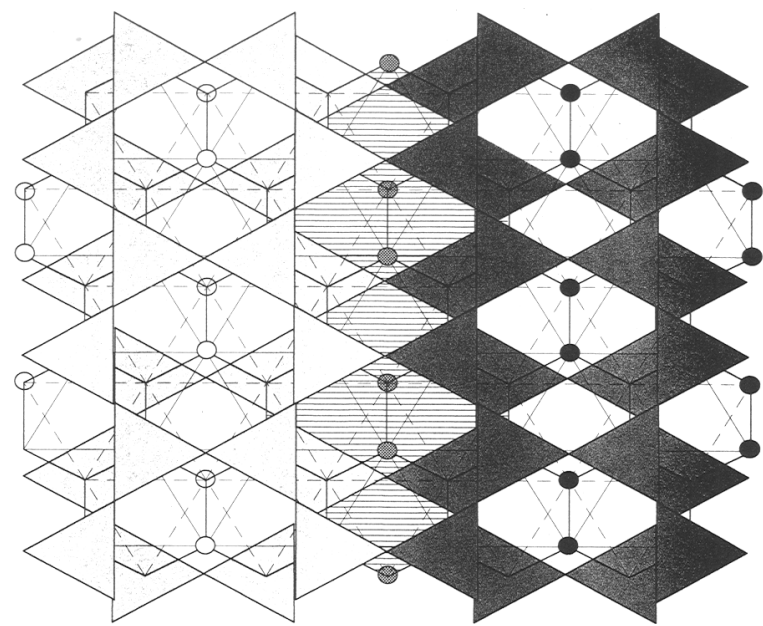

Figure 9. Lateral attachment of two $M$ layers with the same orientation ( $\mathrm{Z}$ stacking vector $33_{0}$ ) preserving the arrangement of the $(\mathrm{OH}, \mathrm{F})$ groups. The octahedra along the boundary are crossed with horizontal lines; open and close circles represent the $(\mathrm{OH}, \mathrm{F})$ positions in the two layers; gray circles correspond to $(\mathrm{OH}, \mathrm{F})$ groups at the boundary. The two $\mathrm{M}$ layers that were originally separated are shown by a different shading of the $T$ sheets. Each of the three sheets $(T-O-T)$ is continuous across the interface. The resulting $M$ layer cannot be distinguished from a single as-grown layer with the same orientation.

homogeneous polytypes belonging to subfamily $B$ would require the introduction of layers with $(2 n+1)$ $\times 60^{\circ}$ orientation into a subfamily-B homogeneous polytype. The probability for a certain polytype to be formed through coalescence of two crystallites is directly related to the frequency of occurrence of the two subfamilies. If $\mathrm{P}_{\mathrm{BS}}(\mathrm{X})$ is the probability of a basic structure to belong to a subfamily $(X)$, and $P_{N L}(Y)$ is the probability of introducing a new layer with an orientation corresponding to a subfamily (Y), the probability $\mathrm{P}$ to form a polytype $(\mathrm{X}, \mathrm{Y})$ is $\mathrm{P}_{\mathrm{BS}}(\mathrm{X}) \times \mathrm{P}_{\mathrm{NL}}(\mathrm{Y})$. Acknowledging that $\mathrm{P}(\mathrm{A})>\mathrm{P}(\mathrm{B})$, it follows that $\mathrm{P}_{\mathrm{BS}}(\mathrm{A}) \times \mathrm{P}_{\mathrm{NL}}(\mathrm{A})>\left[\mathrm{P}_{\mathrm{BS}}(\mathrm{A}) \times \mathrm{P}_{\mathrm{NL}}(\mathrm{B}), \mathrm{P}_{\mathrm{BS}}(\mathrm{B}) \times\right.$ $\left.P_{\mathrm{NL}}(\mathrm{A})\right]>\mathrm{P}_{\mathrm{BS}}(\mathrm{B}) \times \mathrm{P}_{\mathrm{NL}}(\mathrm{B})$, which well agrees with the fact that no subfamily-B inhomogeneous polytypes have been found to date.

\section{Mechanism of stacking sequence modification}

The stacking of layers in polytypic substances must preserve the structural continuity between adjacent layers. For micas, the presence of interlayer cations requires that the centers of tetrahedral rings of adjacent $M$ layers superpose in the (001) projection (Figure 5a). When $\Omega$ and $\Xi$ components interact, the lowermost layer(s) of $\Xi$ protrudes over the top-most layer of $\Omega$ (Figure 6). If the arrangement of the $(\mathrm{OH}, \mathrm{F})$ groups in the two components does not match at the interface, the two tetrahedral rings do not superimpose. If this is the case, such mismatched $M$ layers are called a shifted pair. The amount of the mismatch in a shifted pair

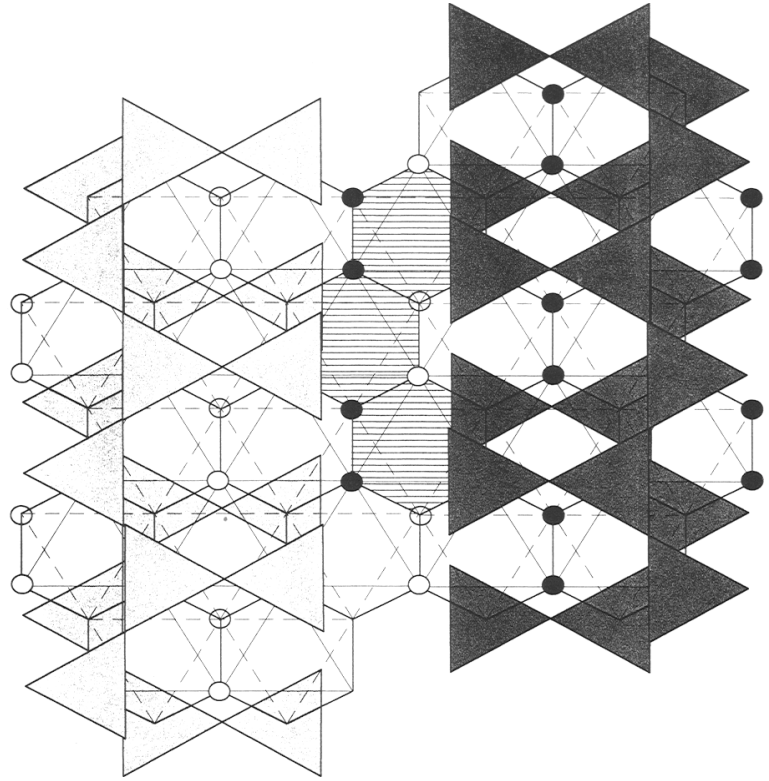

Figure 10. Lateral attachment of two $M$ layers with the same orientation ( $\mathrm{Z}$ stacking vector $33_{0}$ ) but with altered arrangement of the $(\mathrm{OH}, \mathrm{F})$ groups (same drawing conventions as in Figure 9). Two (OH,F) positions from each adjacent layer occur in each octahedron crossed with horizontal lines. The two $T$ sheets are not connected at the boundary. Open circles and close circles correspond to the $I$ positions of each $M$ layer. They do not match across the boundary but are displaced by $|b| / 3$.

depends upon the relative rotation between $\Omega$ and $\Xi$ and, because of the layer stagger, also on the number of layers interacting. Various cases of lateral interaction of two components are possible (Figures 9-13). $\Omega$ and $\Xi$ can be related by six different relative rotations. For a $2 n \times 60^{\circ}$ rotation, either a parallel growth (Figure 9) or a twin according to the (110) or (1.10) law (Figure 10) arises. In both cases, $\Omega$ and $\Xi$ may be related in two possible ways. In one case, the arrangement of the $(\mathrm{OH}, \mathrm{F})$ groups is kept unchanged along the boundary (Figure 9); the two $T$ sheets above and below the $O$ sheet continue across the boundary and the interlayer-cation positions match. In the other case, the octahedra across boundary have in common four $(\mathrm{OH}, \mathrm{F})$ groups (two from each of $\Omega$ and $\Xi$ ) (Figure 10). The two $T$ sheets above and below the $O$ sheet are disconnected across the boundary, as in the case of screw dislocations in polytypes with the $2 n \times 60^{\circ}$ rotations between layers (Baronnet and Amouric, 1986) and the interlayer-cation ( $I$ ) positions have a mismatch of $|b| / 3$. For a $(2 n+1) \times 60^{\circ}$ rotation, a twin according to the (100), (130), or (130) law arises. In this case, the interlayer-cation positions of $\Omega$ and $\Xi$ can never match. The $O$ sheet at the boundary is highly distorted and the two $T$ sheets are disconnected, and the mismatch of the interlayer-cation positions is $|a| / 3$ (Figure 11). 


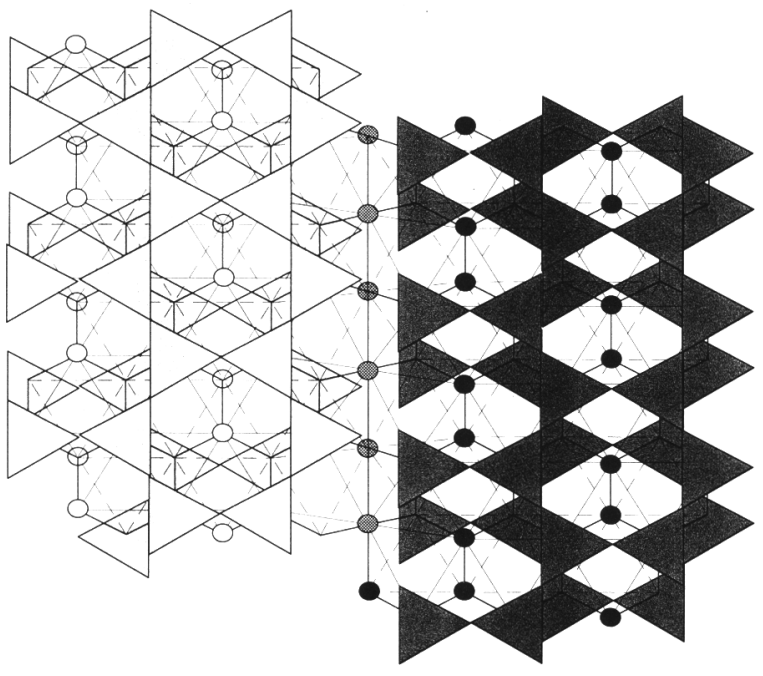

Figure 11. Lateral attachment of two $M$ layers with opposite orientation ( $\mathrm{Z}$ stacking vector $33_{0}$ and $66_{0}$ ) (same drawing conventions as in Figure 9). At the interface, the octahedra from each of the two $M$ layers have opposite slant and the coordination of the corresponding cations is highly distorted. The polyhedra shown in the figure are drawn by simply juxtaposing the two $M$ layers, but the actual coordination at the interface corresponds to more distorted polyhedra.

Where one layer of $\Xi$ protrudes above $\Omega$, the growth of $\Xi$ is unaltered only if a shifted pair is avoided, otherwise the corresponding mismatch must be compensated. A possible, but unlikely compensation of an $|a| / 3$ mismatch is a $C S^{I I}$ at the $O$ sheet. This mechanism would require the slip of both halves of an $M$ layer in opposite directions to avoid forming tri-

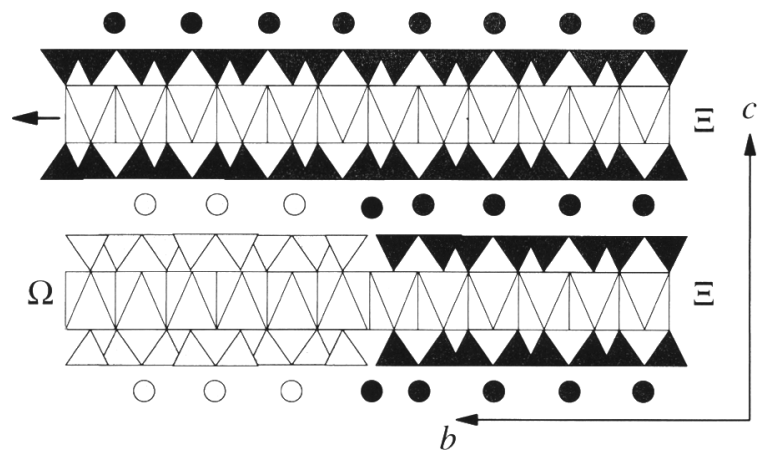

Figure 12. Projection along the [100] direction showing two $M$ layers laterally attached with a relative rotation by $180^{\circ}$ and the next layer growing above. $\Omega$ and $\Xi$ components are shown by different shading of the tetrahedra and interlayer cations. At the boundary, the $O$ sheets have opposite slant and the two $T$ sheets are disconnected. The interlayer cation is shown by a black circle (its position is slightly moved from the ideal, because of the $|a| / 3$ mismatch). The second layer growing from $\Xi$ can cover the surface of $\Omega$ with a distortion of the lower $T$ sheets, realized, e.g., through a reduction of the ditrigonal rotation of the tetrahedra at the boundary. The arrow gives the growth direction of the upper layer.
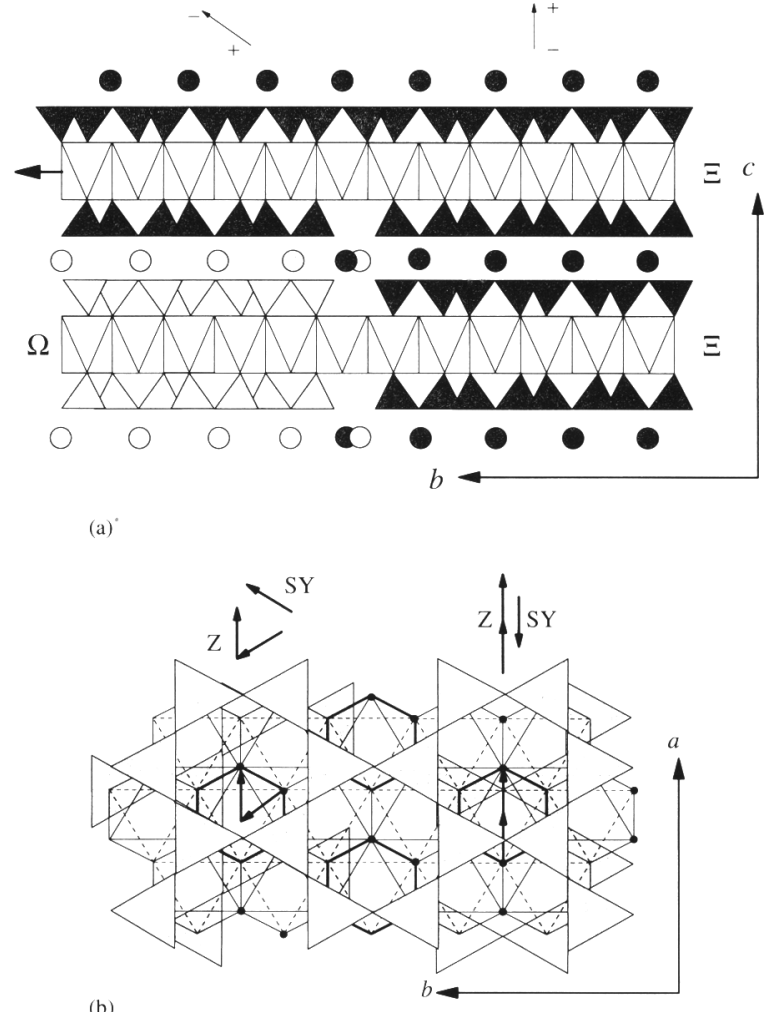

(b)

Figure 13. (a) Projection along the $[\overline{100}]$ direction showing two $M$ layers laterally attached, and with the same orientation, but with a relative shift that alters the $(\mathrm{OH}, \mathrm{F})$ arrangement at the boundary. The $O$ sheets are continuous, whereas the $T$ sheets are discontinuous. In addition, the mismatch of the interlayer position across the protruding $\Xi$ layer is $|b| / 3$, owing to the shifted pair. The interlayer cations of both components are shown at the ideal positions (in projection) along the boundary. The $O$ sheet and the upper $T$ sheet of the protruding $\Xi$ layer grow unaltered. As a consequence, the SY vector of the protruding $\Xi$ layer is rotated by $120^{\circ}$ compared to the orientation in the bulk $\Xi$ ( + and - indicate positions closer to and farther from the observer, respectively). (b) (001) projection of the protruding layer ( $\Xi$ component) in (a). The lower $T$ sheet is discontinuous because of the $|b| / 3$ mismatch. The arrangement of the upper $(\mathrm{OH}, \mathrm{F})$ groups is unchanged across the boundary, but that of the lower groups is modified. The $M 1$ octahedra are transformed into $M 2$ octahedra when $\Xi$ covers $\Omega$; therefore, for meso- and heterooctahedral micas, at the boundary the M1 layer is transformed into an M2 layer and the $Z$ vectors change from $33_{0}$ to $53_{0}$. The vector sum corresponds to the SY vector. Both $Z$ and SY stacking vectors are shown in the upper portion of (b). Z vectors clearly show the change in the layer symmetry, whereas the SY vectors only show a $120^{\circ}$ rotation.

gonal prisms, and would simply propagate the $|a| / 3$ mismatch to the opposite side of the M layer. The $|a| /$ 3 mismatch is more simply accommodated through a local reduction of the ditrigonal rotation of the tetrahedral rings or through a local elastic deformation (Figure 12). These adjustments do not modify the stacking vector of the growing layer. On the contrary, 


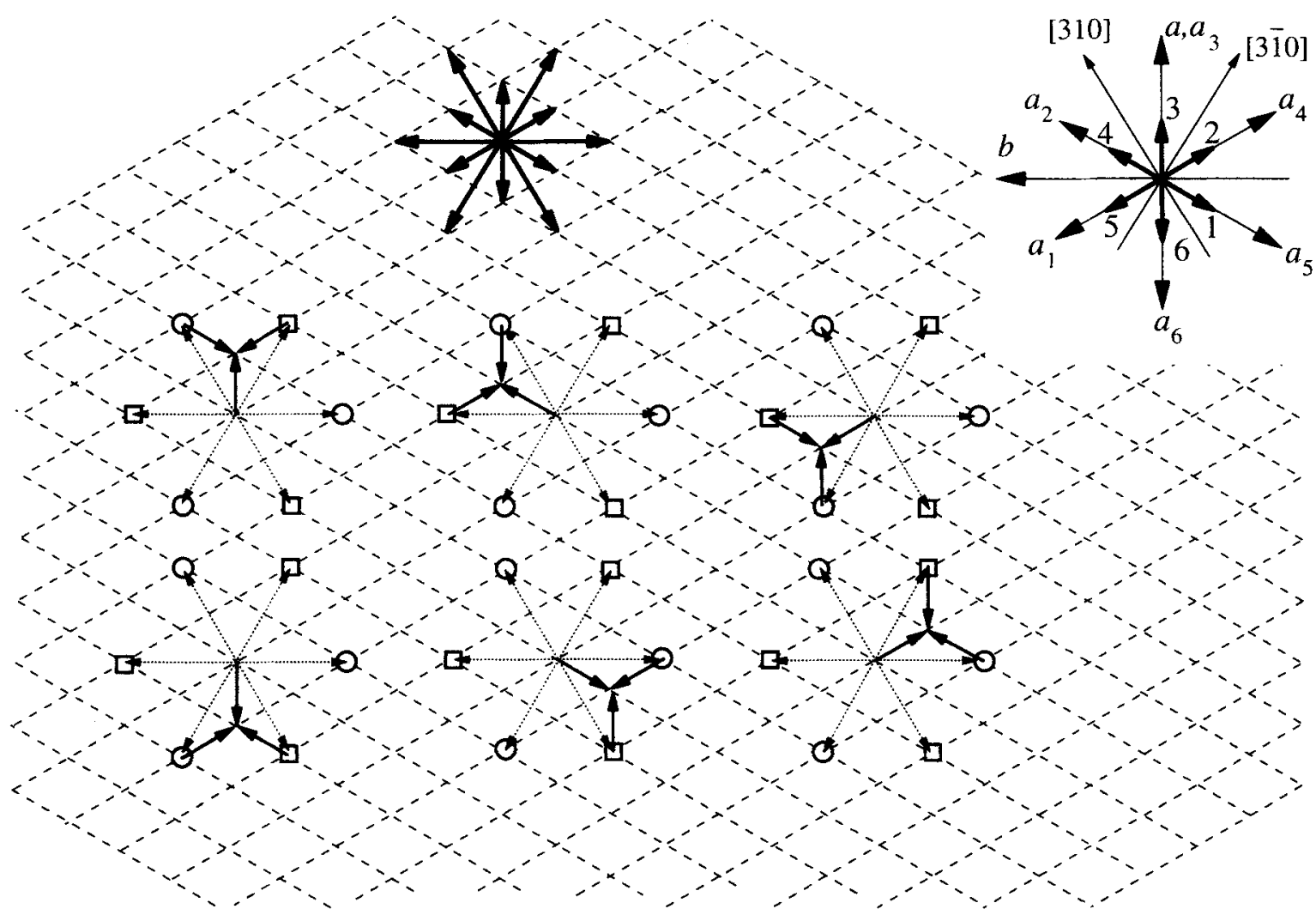

Figure 14. Vector scheme showing the modification of a $\mathrm{Z}$ vector (which gives the absolute orientation of a half $\mathrm{M}$ layer) following a $|b| / 3$ displacement. The intersections of the dashed grid in the background give the possible positions of interlayer $(I)$ cations: the 12 mismatch vectors in the upper part of the figure correspond to the (001) projection of vectors connecting the $I$ position determined by the two tetrahedral rings of a shifted pair (the six short vectors correspond to $|a| / 3$ and the six long ones to $|b| / 3$ ). In the lower part of the figure, the transformation rules for the $Z$ vectors owing to a $|b| / 3$ mismatch (dotted arrows) are given for the six orientations of the original $Z$ vector ( $c f$., inset on the top-right, where coordinate vectors and $Z$ vectors are shown). The figure is drawn for a structural adjustment in the lower half layer of an $M$ layer, i.e., corresponding to the insertion of a growth defect in an $M$ layer growing onto a boundary. Therefore, the head of the $Z$ vector, representing the origin of the $O$ sheet, is fixed. The original position of the $I$ cation below the $M$ layer, represented by the tail of the $Z$ vector, is at the intersection of the six $|b| / 3$ vectors (dotted). By applying any of the $|b| / 3$ translations, the tail of the $Z$ vector moves into one of two possible, translationally independent positions shown by open circles $(1 / 6[310],-1 / 6[3 \overline{1} 0],-1 / 3[010])$ and open squares $(-1 / 6[310], 1 / 6[3 \overline{1} 0], 1 / 3[010])$, respectively. In case of a structural adjustment in the upper half layer of an $\mathrm{M}$ layer, i.e., corresponding to the occurrence of a $\mathrm{CS}^{\mathrm{I}}$ into an $\mathrm{M}$ layer of a small platelet or crystal covered by a larger spiral, the tail of the $\mathrm{Z}$ vector represents the origin of the $O$ sheet and is unchanged, whereas the head represents the interlayer position and is moved. This situation can be described by the same figure, with tail and head of the $Z$ vectors exchanged.

a $|b| / 3$ mismatch requires a structural adjustment consisting either in a growth defect or a crystallographic slip.

A growth defect which is allowed to compensate for the tetrahedra discontinuity at a $\Xi-\Omega$ boundary with a $|b| / 3$ mismatch, is related to the loss of a row of tetrahedra from the lower tetrahedral sheet (Figure 13a). The overlying $O$ and $T$ sheets of this $M$ layer are not affected. The net result is shown in Figure 13b, which is the (001) projection of the upper layer in Figure 13a. The octahedra that, in the bulk of $\Xi$ are trans, are shown by thick lines. After reaching the discontinuity below (Figure 14), the lower $(\mathrm{OH}, \mathrm{F})$ groups are displaced by $|b| / 3$ (in Figure $13 b$, the displacement is shown along the $[3 \overline{10}$ ] direction), but the position of the upper $(\mathrm{OH}, \mathrm{F})$ groups does not change. The growth of $\Xi$ over $\Omega$ has thus two consequences: 1) although the distribution of cations in the octahedra did not change, the position of the $(\mathrm{OH}, \mathrm{F})$ groups in the lower plane of apical oxygen atoms did (as well as the tetrahedra bonded to them); 2) the lower $Z$ vector was rotated by $120^{\circ}$ and the $Z$ symbols have changed from $33_{0}$ to $53_{0}$. $Z$ vectors and $S Y$ vectors are indicated in Figure 13b, from which it is clear that, for meso- or hetero-octahedral micas, the M1 layer was transformed into an M2 layer after reaching the $\Xi-\Omega$ boundary. The layer growing above the $\Xi-\Omega$ boundary is thus partially M1 (before reaching the boundary) and partially M2 (after reaching the boundary), but the $S Y$ vectors simply show the existence of 
Table 2. Transformation rules for the orientation of the $Z$ vector due to a $|b| / 3$ mismatch of the interlayer cation position along each of the six possible directions.

\begin{tabular}{lcc}
\hline & \multicolumn{2}{c}{ Shift } \\
\cline { 2 - 3 } Orientation parity & $-1 / 6[310], 1 / 6[3 \overline{3} 0]$, & $1 / 6[310],-1 / 6[3 \overline{1} 0]$, \\
$1 / 3[010]$ & $-1 / 3[010]$ \\
\hline Odd & $\mathrm{i}+2$ & $\mathrm{i}-2$ \\
Even & $\mathrm{i}-2$ & $\mathrm{i}+2$ \\
\hline
\end{tabular}

two stacking vectors rotated $\pm 120^{\circ}$ within the same layer, This phenomenon was observed in the synthetic fluorphlogopite studied by Bloss et al. (1963), who reported a variation of the layer-stagger direction within different portions of the same layer.

A similar compensation of a $|b| / 3$ mismatch and, as a consequence, an M1 $\rightarrow$ M2 transformation of the layer type can also occur through a crystallographic slip of the first sort $\left(\mathrm{CS}^{\mathrm{I}}\right)$. The structural adjustment does not necessarily occur immediately, especially if the density of interlayer cations close to the boundary is low. The protrusion of $\Xi$ over $\Omega$, and the corresponding increase in the number of interlayer cations in the mismatched position, strengthens the driving force for structural adjustment. The stress resulting from the shifted pair, together with the physical continuity between each of the two layers of the shifted pair and the bulk of the two components $\Xi$ and $\Omega$, causes a CSI in one of the two layers. The CS $^{I}$ would require the breaking of bonds between tetrahedra and has thus a low probability of occurrence. However, if an entire $T$ sheet is simultaneously displaced, no such breaking of bonds is necessary. A CS ${ }^{I}$ in the upper layer of the shifted pair (lowest layer protruding from $\Xi)$ is unlikely to involve an entire atomic plane because of the physical continuity with a large matrix. In addition, the result would be undistinguishable from that of the growth defect described above. The CSI can instead involve the entire $T$ sheet of the lower layer of the shifted pair (top-most layer of $\Omega$ ) if $\Omega$ is significantly smaller than $\Xi$, as for example, in the case of spiral-crystal interaction (platelet deposition). Such a slip is favored by a crystal-chemical configuration with strong bonds in the interlayer and, at the same time, a weak destabilized $O$ sheet, such as that found in oxybiotites and titaniferous (oxy)biotities. As a matter of fact, most of the inhomogeneous polytypes in Li-poor trioctahedral micas have this composition (Ross et al., 1966; Borutskiy et al., 1987; Zhukhlistov et al., 1993; Bigi and Brigatti, 1994; Nespolo and Takeda, 1999; Kogure and Nespolo, 1999a).

The modification of the $Z$ vector is shown in Figure 14 for all six orientations. The six vectors corresponding to six possible $|b| / 3$ shifts are shown by dotted lines (not to be confused with the dashed lines, for the underlying grid); their common point is the origin of the $\mathrm{Z}$ vector before the structural adjustment. These ad- justments modify the orientations of the $\mathrm{Z}$ vector in one of two possible orientations having a different origin (shown by circle and square, respectively) but a common end. Transformation rules for $\mathrm{Z}$ symbols are given in Table 2. The M1 layer where the structural adjustment occurred is transformed into an M2 layer whose $Z$ vector has changed from $i_{0}$ to either $i \pm 2$, $\mathrm{i}_{0}$ (growth defect) or $\mathrm{i}, \mathrm{i} \pm 2_{0}\left(\mathrm{CS}^{\mathrm{I}}\right)$, which are translationally equivalent to either $i-2, i-2_{0}$ or $i+2, i$ +2 (Figure 15).

\section{EXAMPLES OF APPLICATION OF THE PERTURBATIVE MODEL TO THE RUIZ PEAK POLYTYPES}

The titaniferous oxybiotite from the Ruiz Peak rhyodacite ash flow, Jemez Mountains, New Mexico (Smith et al., 1961; Takeda and Ross, 1975; Bailey and Smith, 1978; Ohta et al., 1982) was the subject of extensive X-ray and HRTEM studies revealing not only all the three basic structures, but also the longest and most complex inhomogeneous polytypes reported to date (Ross et al., 1966; Nespolo and Takeda, 1999; Kogure and Nespolo, 1999a), a large number of twins (about one third of the crystals; Ross and Wones, 1965), and also some allotwins (Nespolo et al., 2000). The composition, rich in ferric iron and titanium, is close to the upper compositional stability limit for oxybiotites. The numerous planar defects are interpreted as early stages of decomposition (Kogure and Nespolo, 2001).

In the volcanic fumarole where it was formed, settling of thin crystals onto the surface of a larger crystal, with consequent spiral-crystal or crystal-crystal interaction, is the mechanism by which at least some of the inhomogeneous polytypes reported so far were formed (Kogure and Nespolo, 1999a). The interpretation of the stacking sequence of three inhomogeneous polytypes from the Ruiz Peak oxybiotite is presented here. Only the unfaulted stacking sequences of the three basic structures are used as components. The inclusion of stacking faults in the basic structures would add flexibility to the model and give more simple routes to explain the observed stacking sequences, but to illustrate the method in the most direct way, the presence of stacking faults is not considered in these examples. Two polytypes were identified by PID analysis (periodic intensity distribution: Takeda, 1967; Takeda and Ross, 1995; Nespolo et al., 1999a) of the X-ray diffraction patterns (Ross et al., 1966), whereas the third polytype was determined by HRTEM (Kogure and Nespolo, 1999a). Both techniques reveal the stacking sequence as described within the homo-octahedral approximation (i.e., assuming all M1 layers).

\section{Example 1. $8 A_{12}$ polytype}

The $8 A_{12}$ polytype was found by Ross et al. (1966) and described by RTW symbols as 22020002 . The 

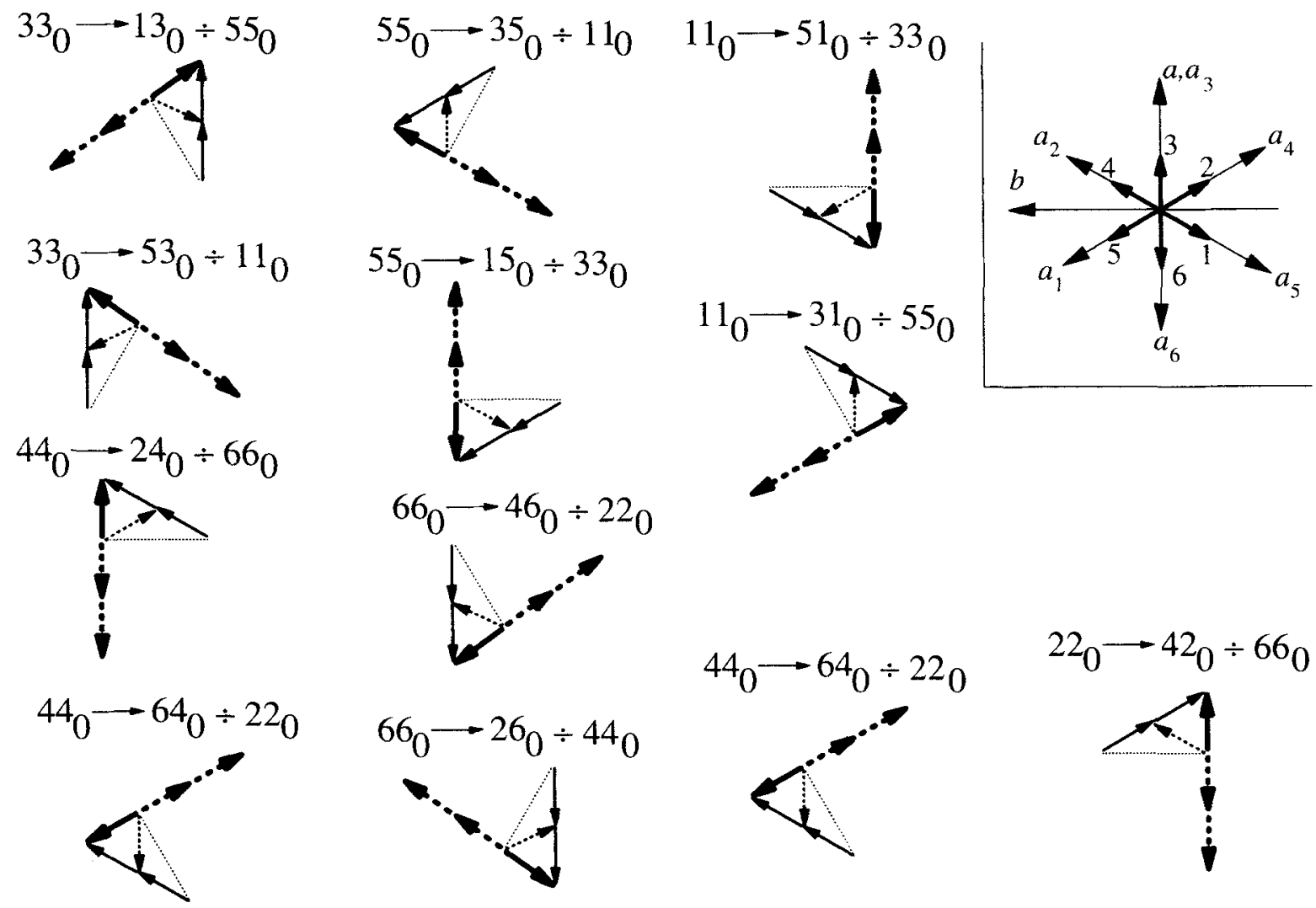

Figure 15. Transformation rules for an $\mathrm{M} 1 \rightarrow \mathrm{M} 2$ layer transformation obtained through a $|b| / 3$ shift of the lower half-layer (structural adjustment through insertion of a growth defect). Solid thin vector pairs are pairs of $Z$ vectors before structural adjustment. Dotted thin lines are directions of the shift. Dotted thin vectors are lower $Z$ vectors after structural adjustment. Solid thick vectors are SY vectors. Dotted thick vectors are pairs of $Z$ vectors as they appear in the homo-octahedral approximation (translationally equivalent to $\mathrm{SY}$ vectors). The symbol " $\mathrm{ii}_{0} \rightarrow \mathrm{ji}_{0} \div \mathrm{kk}_{0}$ " stands for "layer with orientation $\mathrm{ii}_{0}$ transforms to $\mathrm{ji}_{0}$, which, in the homo-octahedral approximation, is equivalent to $\mathbf{k k}_{0}$ ". A similar figure can be drawn for the case of a structural adjustment obtained through a CS , altering the second $Z$ vector (upper half of the $M$ layer) instead of the first one. Inset on the right upper corner: $Z$ vectors and coordinate axes.

shortened $\mathrm{Z}$ symbols are 64224444 , and the $\mathrm{Z}$ vectors are shown in Figure 16. The observed stacking sequence is interpreted as relating to coalescence of a $1 M$ (E) and a $3 T(\Omega)$ crystal. The lateral interaction through their $\mathrm{S}$ faces produced a screw dislocation around which five layers from $1 M$ and three layers from $3 T$ grew on each other. Where the lowermost of the five $1 M$ layers started to protrude onto the surface of the $3 T$ crystal, a shifted pair appeared corresponding to $a+b / 3$ mismatch along the [310] direction. As a consequence, a structural adjustment occurred during growth, as described in Figure 10 . The $+b / 3$ shift of the interlayer position along the [310] direction corresponds to a rotation of the lowest $\mathrm{Z}$ vector from orientation 4 to orientation 6 . Thus, the first of the five $1 M$ layers $(\Psi)$ changed from M1 to M2 and the $Z$ symbol changed from $44_{0}$ to $64_{0}$, which in the homo-octahedral approximation is equivalent to $22_{0}$. The three $3 T$ layers protruding onto the surface of the $1 M$ crystal produced a shifted pair corresponding to a $-a / 3$ mismatch along the [100] direction, but this mis- match can be compensated without modification of the $\mathrm{Z}$ vector, as shown above.

\section{Example 2. $10 \mathrm{~A}_{3}$ polytype}

The $10 A_{3}$ polytype was found by Ross and Wones (1965). The stacking sequence as determined by Ross et al. (1966) in RTW symbols is 2200222222. The shortened $Z$ symbols are 51333513513 . This polytype belongs to the structural series $3 T$. It can be explained by coalescence and lateral attachment of two $3 T$ crystallites ( $\Xi$ and $\Omega$ ), with formation of a screw dislocation around which cooperative growth started. Seven layers from $\Xi$ protrude onto the surface of $\Omega$ and three layers from $\Omega$ protrude onto the surface of $\Xi$ (Figure 17). Where the seven layers from $\Xi$ start to protrude, a shifted pair corresponding to a $-b / 3$ mismatch along [010] occurs. The lowest half layer compensated this mismatch by insertion of a growth defect. This defect changed the corresponding $Z$ vector from 1 to 5 . Thus, the first of the seven layers $(\Psi)$ changed from $M 1$ to 


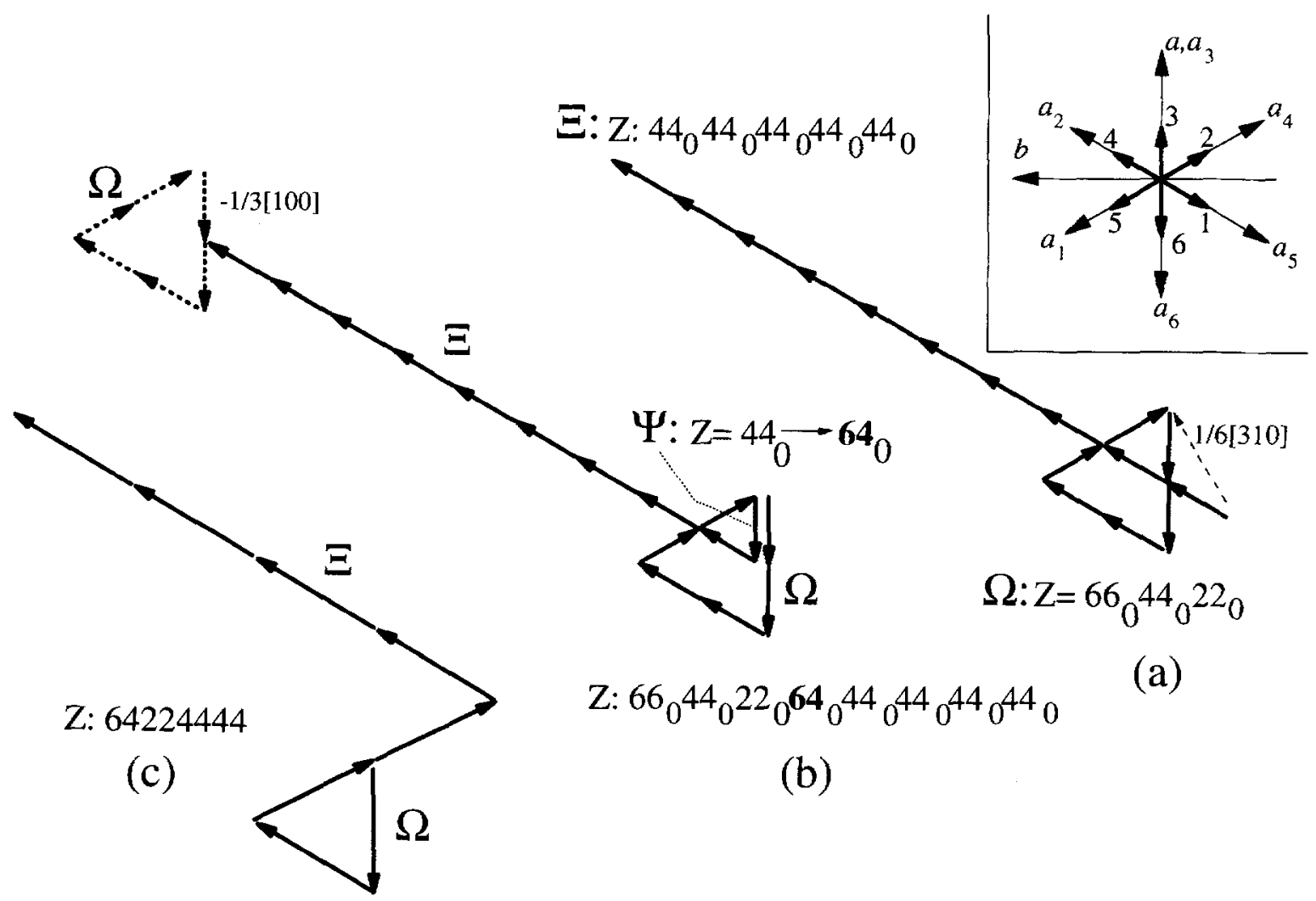

Figure 16. Interpretation of the formation mechanism of the $8 A_{12}$ polytype. A $1 M(\Xi)$ and a $3 T(\Omega)$ crystal coalesce and laterally attach, producing a screw dislocation around which cooperative growth starts (cf., Figure 8). A shifted pair is created, corresponding (a) to $a+b / 3$ mismatch along [310], and the lowermost half layer is modified by the introduction of a growth defect. This corresponds to a $120^{\circ}$ rotation of the corresponding $Z$ vector, which changes from orientation 4 to orientation 6 . The corresponding layer $(\Psi)$ changes from $M 1$ to $M 2$ and the resulting $Z$ symbols are $64_{0}$, which in the homo-octahedral approximation are equivalent to $22_{0}$ (b). From $\Omega$, three layers protrude onto the surface of $\Xi$ and a $-a / 3$ shift along the [100] direction is produced, which can be compensated without alteration of the corresponding $Z$ vector (b). The resulting stacking vectors ( $Z$ vectors) are as in (b), where the bold digits indicate the two $Z$ vectors of the $M 2$ layer. In the homo-octahedral approximation the $Z$ vectors appear as in (c). Long vectors are complete $Z$ vectors for M1 layers, (length $2|a| / 3$ ). Short vectors are $\mathrm{Z}$ vectors for each half layer (length $|a| / 3$ ). Inset on the right upper corner: $Z$ vectors and coordinate axes.

$\mathrm{M} 2$ and the $\mathrm{Z}$ vectors changed from $55_{0}$ to $51_{0}$, which in the homo-octahedral approximation is equivalent to $33_{0}$. At the same time, the three layers protruding from $\Omega$ onto the surface of $\Xi$ produced a shifted pair corresponding to a $-a / 3$ mismatch along [100], but this mismatch can be compensated without modification of the $Z$ vectors. The resulting stacking sequence corresponds to $Z$ vectors $55_{0} 11_{0} 33_{0} 51_{0} 33_{0} 55_{0} 11_{0} 33_{0} 55_{0} 11_{0}$.

Although the $10 A_{3}$ polytype is triclinic, it gives a monoclinic diffraction pattern (Ross et al., 1966): this phenomenon is known as "diffraction enhancement of symmetry" (Ito, 1950). Sadanaga and Takeda (1968) showed that the monoclinic character of the diffraction pattern from this polytype is caused by the existence of sets of unit layers, equal in configuration. The restriction on the layer symmetry is that it possesses a two-fold axis, in the direction parallel to the $b$ axis of the crystal. The $\mathrm{M} 2$ layer $51_{0}$ contains as the only symmetry element a two-fold axis, which is located along the $b$ axis of the crystal (for the symmetry rules relating Z symbols see Zvyagin, 1997 and Nespolo et al., 1999a). Thus, the formation mechanism, as involving a growth defect transforming one of the M1 layers into an M2 layer, does not contrast with the diffraction enhancement of symmetry as observed in the diffraction pattern of this polytype.

\section{Example 3. $32 A_{1}$ polytype}

Polytype $32 A_{1}$ belongs to subfamily $\mathrm{A}$ and was found by HRTEM investigation (Kogure and Nespolo, $1999 \mathrm{a}$ ). It is the second longest-period polytype in micas (following the $36 A_{1}$ polytype, found in the same sample) and it has the most complex stacking sequence found thus far in micas. RTW symbols are $2 \overline{2} 22 \overline{2}(2)_{5} \overline{2} 22 \overline{2} 2(2)_{6} \overline{2}(2)_{7} \overline{2} 22$ and shortened $Z$ symbols are $6262424624646264(62462424)_{2}$. This stacking sequence is interpreted as relating to the coalescence of the $3 T$ and $2 M_{1}$ basic structures, so that this polytype 


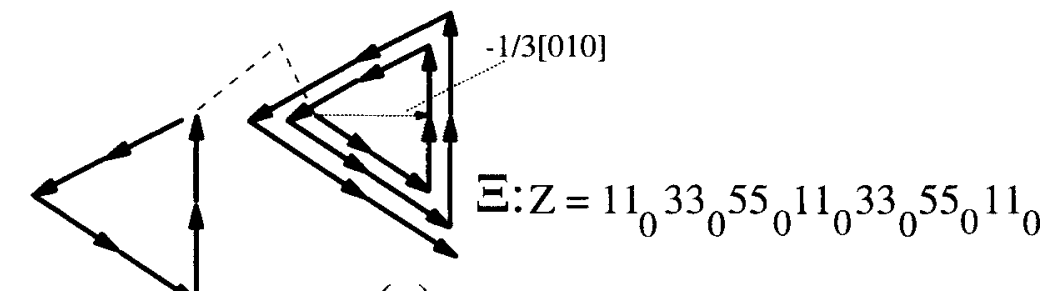

(a)

$\Omega: Z=55_{0} 11_{0} 33_{0}$
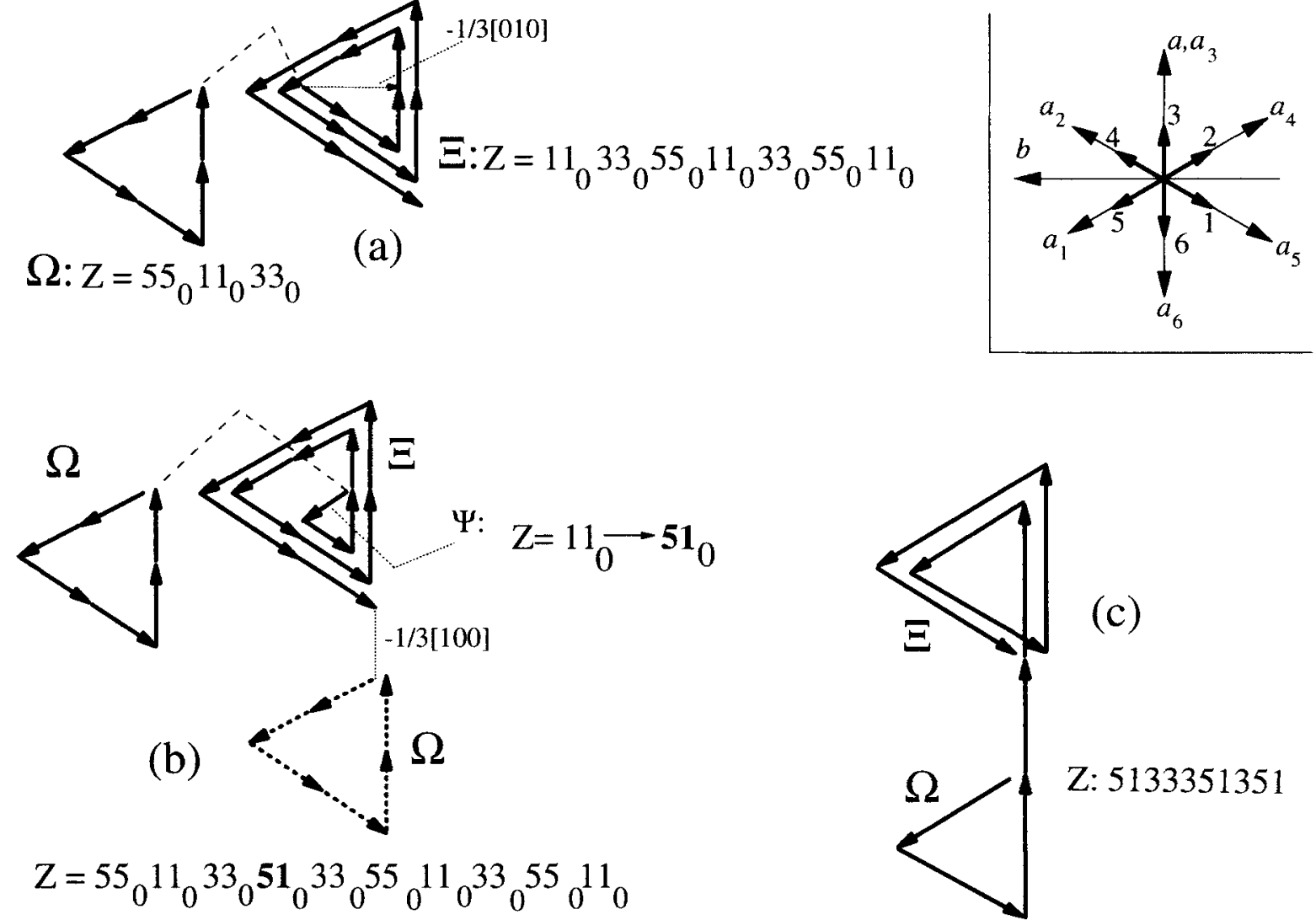

(c)

Figure 17. Interpretation of the formation mechanism of the $10 A_{3}$ polytype. Two $3 T$ crystals ( $\Xi$ and $\Omega$ ) coalesce and laterally attach, producing a screw dislocation around which cooperative growth starts ( $c f$., Figure 8 ). A shifted pair corresponding to $a-b / 3$ mismatch along the [010] direction occurs (a) and the lowermost half layer is modified by the introduction of a growth defect. This corresponds to a $120^{\circ}$ rotation of the corresponding $Z$ vector, which changes from orientation 5 to orientation 1 . The corresponding layer $(\Psi)$ changes from $M 1$ to $M 2$ and the resulting $Z$ symbols are $51_{0}$; in the homo-octahedral approximation they are equivalent to $33_{0}$ (b). From $\Omega$ three layers protrude onto the surface of $\Xi$ and a $-a / 3$ displacement along [100] is produced, which can be compensated without alteration of the corresponding $Z$ vector (b). The resulting $Z$ vectors are as in (b). Graphic conventions same as in Figure 16.

is related to both structural series. The analysis of the possible formation mechanism is complex: to show the mismatches and the structural adjustments, the $(a, b)$ components of $Z$ vectors are given in the space-fixed reference, taking into account that $\pm 2 / 3$ is translationally equivalent to $\mp 1 / 3$.

Two different subperiods are easily recognized, corresponding to layers $1-16$ and layers $17-32$. The latter subperiod contains two additional subperiods (layers 1724 and layers 25-32) which are identical. It thus corresponds to two turns of an eight-layer complex spiral.

The 16-layer subperiod 1-16 can be explained by the coalescence of $3 T$ and $2 M_{1}$ crystals. Layers $1-4$ belong to a $2 M_{1}$ crystal $(\Omega)$, with original orientation $(64)_{2}$, cooperatively growing with a coalesced $3 T$ crystal $(\Xi)$ with orientation (624) 62 (Figure 18a). An eight-layer ledge

Figure 18. Interpretation of the formation mechanism of the $32 A_{\perp}$ polytype. (a) Cooperative growth of a $2 M_{1}(\Omega)$ and a $3 T(\Xi)$ crystal. A 12-layer polytype is formed through two structural adjustments $(\Psi)$, consisting in a growth defect at the boundary $\Omega$ $\Xi$ and a $C^{1}$ at the boundary $\Xi-\Omega$. Both structural adjustments are due to a $-b / 3$ mismatch along [310] and both $o c c u r$ in the $\Xi$ component. (b) 16 layers from the 12-layer polytype $(\Omega)$ formed in (a) cooperatively grow with 16 layers from an eight-layer polytype $(\Xi)$ that was formed by coalescence of a $3 T$ (six layers) and a $2 M_{1}$ (two layers) crystals. The 16 layers protruding from $\Xi$ onto the surface of $\Omega$ produced a shifted pair corresponding again to a $-b / 3$ mismatch along [310], which was compensated by insertion of a $C^{1}$ in the uppermost $(\Psi)$ layer of $\Omega(c)$. The second period of the 32-layer polytype begins with a $+a / 3$ mismatch along [110], which does not need structural adjustment to be compensated. The stacking sequence is shown by means of $\mathrm{Z}$ vectors (length $|a| / 3)$. $\mathrm{Z}$ symbols corresponding to layers in which $C S^{1}$ occurred are marked in bold. 

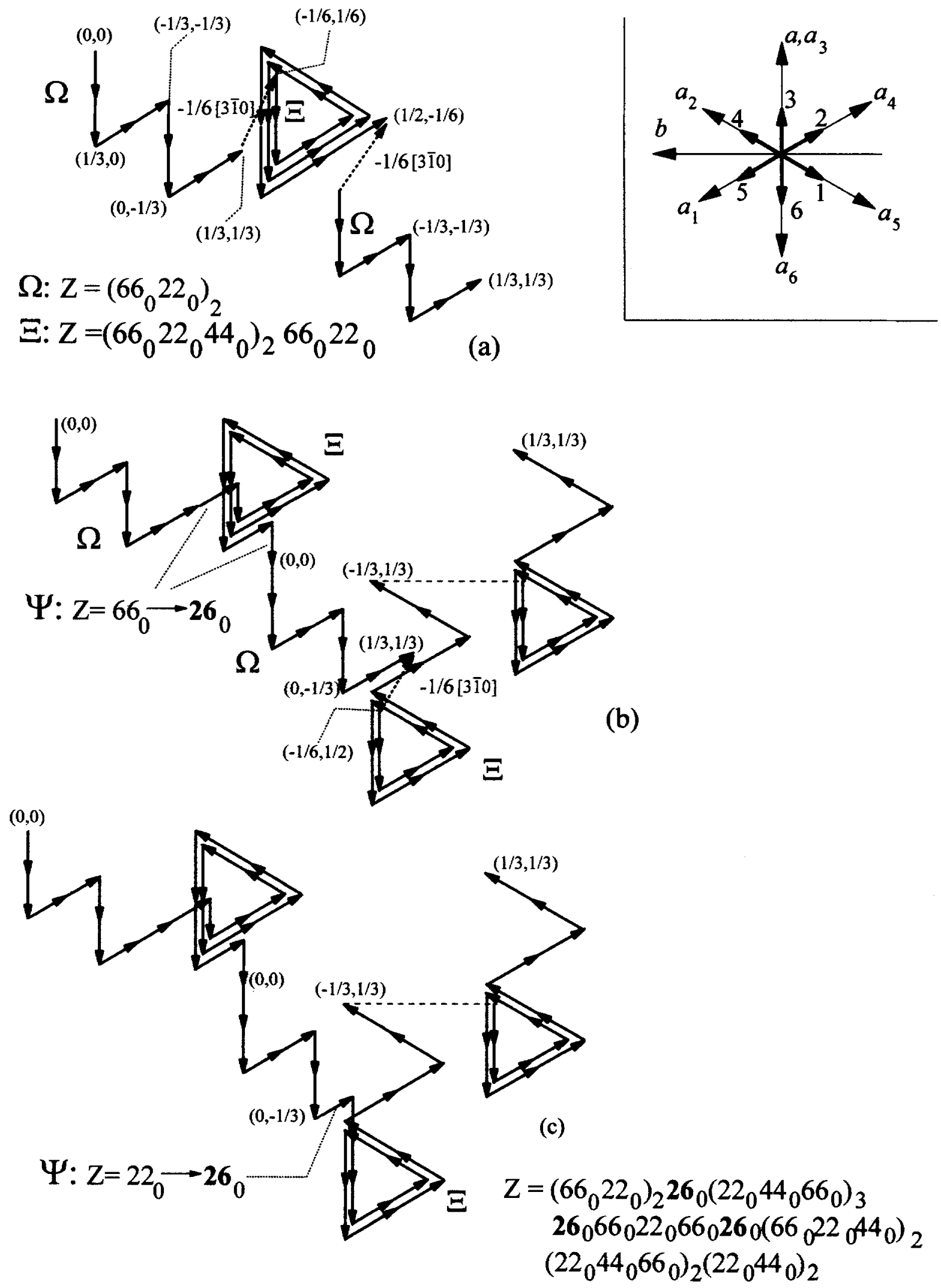
protruded from $\Xi$ onto the surface of $\Omega$. Assuming the origin of the four-layer ledge of $\Omega$ in correspondence of coordinates $(0,0)$, its fourth $Z$ vector ends with coordinates $(1 / 3,1 / 3)$ (Figure $18 a)$. If the origin of the eightlayer $\Xi$ ledge was at $(-1 / 6,1 / 6)$ where it protruded on crystal $\Omega$, the ledge produced a shifted pair with $a-b / 3$ mismatch along the $[3 \overline{1} 0]$ direction. The structural adjustment $(\Psi)$ is seen in the first layer of $\Xi$. Therefore, this adjustment corresponds to a growth defect. The stacking sequence of $\Xi$, expressed by complete $Z$ symbols, changes from $66_{0} 22_{0} 44_{0} 66_{0} 22_{0} 44_{0} 66_{0} 22_{0}$ to $26_{0} 22_{0} 44_{0} 66_{0} 22_{0} 44_{0} 66_{0} 22_{0} ; \mathrm{Z}=26_{0}$ is translationally equivalent to $Z=44_{0}$ (Figure 18a). The uppermost layer of $\Xi$ ends with coordinates $(1 / 2,-1 / 6)$. Four layers from $\Omega$ protrude onto the surface of $\Xi$. The first layer of $\Omega$ has coordinates $(0,0)$, leading again to a mismatch of $-b / 3$, along $[\overline{3} 10]$ direction. This time, the structural adjustment is seen in the uppermost layer $(\Psi)$ of $\Xi$, which undergoes a $C S^{\prime}$, changing its orientation from $Z=22_{0}$ to $Z=26_{0}$ (Figure $18 \mathrm{~b}$ ). This kind of structural adjustment indicates that an entire plane slipped, and that a significant difference in size between the two crystals exists $(\Omega \gg \Xi)$. By means of two structural adjustments (one growth defect and one $C^{I}$, both in $\Xi$ ) a perfect match of the interlayercation positions was achieved and, from this point, a 12-layer polytype begins to grow. The $\mathrm{Z}$ symbols are $66_{0} 22_{0} 66_{0} 22_{0} 26_{0} 22_{0} 44_{0} 66_{0} 22_{0} 44_{0} 66_{0} 26_{0}$, which are equivalent to 626242462464 in the homo-octahedral approximation.

Growth of the 12-layer polytype was then modified by cooperative growth with another crystal. This second crystal (layers 17-32) was an eight-layer polytype formed by interaction of six layers from a $3 T$ crystal and two layers from a $2 M_{1}$ crystal. Two periods (16 layers) of this polytype ( $\Xi$ ) protruded onto the surface of the 12-layer polytype $(\Omega)$, from which a 16-layer sequence protruded onto the surface of $\Xi$. Fixing the coordinates of the origin of the first layer as $(0,0)$, the ledge ends at $(1 / 3,1 / 3)$. If the origin of $\Xi$ is at $(-1 / 6$, $1 / 2$ ), then a mismatch of $-b / 3$ occurs, along the [3피 direction (Figure 18b). The structural adjustment occurs as a $C S^{I}$ in the uppermost layer $(\Psi)$ of $\Omega$, and this is in favor of a significant difference in size between the two crystals ( $\Xi \gg \Omega$ ). The corresponding $\mathrm{Z}$ vector changes from $22_{0}$ to $26_{0}$, translationally equivalent to $44_{0}$. The uppermost layer of $\Xi$ (which is also the uppermost layer of the 32-layer polytype) ends with coordinates $(1 / 3,1 / 3)$ and $a+a / 3$ mismatch along [110] arises with respect to the lowermost layer of $\Omega$, i.e., the first layer of the second repeat of the 32-layer polytype, with coordinates $(0,0)$. As seen above, this mismatch is compensated without need of a structural adjustment. Complete $\mathrm{Z}$ symbols are given in Figure $18 \mathrm{c}$, where the vectors of the layers in which $\mathrm{CS}^{\mathrm{I}} \mathrm{oc}$ curred are marked by bold characters.

\section{DISCUSSION}

Growth defects and $\mathrm{CS}^{\mathrm{I}}$ modify the location of the $M 1$ site and can be described as $\pm 120^{\circ}$ rotations of the SY vector. Although this effect is unimportant for homo-octahedral micas, in meso- and hetero-octahedral micas it transforms an M1 layer into an M2 layer. M2 layers were found both in trioctahedral and in dioctahedral micas, but the coexistence of $\mathrm{M} 1$ and $\mathrm{M} 2$ layers in an inhomogeneous polytype has not been proved as yet. The occupation of the cationic sites in the $O$ sheet is modified, but this change may be difficult to extract from a structure refinement. Because of the physical deformation and bending of the crystals, the quality of a diffraction pattern from mica is often poor, with oval-shaped, broad, and streaked reflections. The poor quality of diffraction is a severe obstacle to structure refinement. To date, the only structure refinement of an inhomogeneous polytype $\left(3 M_{2}\right)$ was performed by Rule et al. (1987), with a final $R$ factor of $12 \%$. The quality of the refinement is clearly insufficient to distinguish the possible presence of an M2 layer (furthermore, this is a mixed-rotation polytype, in which the $(2 n+1) \times 60^{\circ}$ rotation does not involve an M1 $\rightarrow$ M2 layer transformation, as in the case of subfamily-A polytypes). However, even with crystals of better quality, the presence of an M2 layer may escape the structural investigation, if the investigator does not suspect its presence.

Consider a hypothetical $N$-layer meso-octahedral polytype, of any stacking sequence, with biotitic composition, and suppose, for simplicity, that there are two $\mathrm{Mg}$ and one $\mathrm{Fe}^{2+}$ ions in the $O$ sheet. Suppose also that one layer is of M2 type, and the remaining $N-$ 1 layers are of M1 type. The occupation of the cation sites in the $O$ sheet is described as: M1 layers: $M 1=$ $(1-x) \mathrm{Fe}+x \mathrm{Mg} ; M 2, M 3: 0.5 x \mathrm{Fe}+(1-0.5 x) \mathrm{Mg}$; M2 layer: $M 2=(1-x) \mathrm{Fe}+x \mathrm{Mg} ; M 1, M 3: 0.5 x \mathrm{Fe}$ $+(1-0.5 x) \mathrm{Mg}$. If the value of $x$ is far from $2 / 3$, the presence of the M2 layer should, in principle, be revealed by a structure refinement. However with the approach of $x$ to $2 / 3$ (where the difference between M1 and M2 disappears), the distinction between $N$ layers of type $\mathrm{M} 1$ and $(N-1)$ layers of type M1 plus one layer of type M2 becomes difficult. The presence of an M2 layer may be mistaken for disorder in the cation distribution. Reliable structure refinements of inhomogeneous polytypes, with particular attention to the cationic distribution in the $O$ sheet, are highly desirable.

\section{ACKNOWLEDGMENTS}

This paper is dedicated to Prof. Boris B. Zvyagin on the occasion of his 80th birthday, for his pioneering work on the polytypism of layer silicates. This research was partly developed at the Mineralogical Institute, University of Tokyo, during a Doctoral program (1995-98) supported by the Japanese Ministry of Education, Science and Culture. The author wishes to express his deep gratitude to I. Sunagawa (Yamanashi 
Institute of Gemology and Jewelry Arts) for helpful discussions and for critical reading of the manuscript. He is also indebted to $\mathrm{H}$. Takeda (Chiba Institute of Technology), J.F Banfield (University of Wisconsin-Madison), Y. Takéuchi (Nihon University, Tokyo), T. Kogure (University of Tokyo), G. Ferraris (University of Torino), B.B. Zvyagin (IGEM, RAS, Moscow), and M. Ross (U.S. Geological Survey) for several helpful discussions, and to S. Guggenheim (University of Illinois, Chicago), S. Ďurovič (Slovak Academy of Sciences, Bratislava), D. Noe (John Hopkins University), and B. Grobety (University of Fribourg) for reviewing the manuscript. J. Jedwab (University of Brussels) and D. Yu. Pushcharovsky (University of Moscow) kindly provided consultation material. M. Miyamoto (University of Tokyo) and Y. Watanabe (Teikyo Heisei University) are acknowledged for their constant encouragement.

\section{REFERENCES}

Amelinckx, S. and Dekeyser, W. (1953) Le Polytypisme des Minéraux Micacés et Argileux. Premiére partie: Observations et leaurs interprétations. Comptes Redus de la XIX session Congrès Geologique International, Comité International pour l'Étude des Argiles, Alger, fascicule XVIII, $1-22$.

Axelrod, J.M. and Grimaldi, F.S. (1949) Muscovite with small optic axial angle. American Mineralogist, 34, 559-572.

Bailey, R.A. and Smith, R.L. (1978) Volcanic Geology of the Jemez Mountains, New Mexico. New Mexico Bureau of Mines, Socorro, New Mexico, Circular 163, 184-196.

Bailey, S.W. (1984) Review of cation ordering in micas. Clays and Clay Minerals, 32, 81-92.

Bailey, S.W. and Christie, O.H.J. (1978) Three-layer monoclinic lepidolite from Tørdal, Norway. American Mineral ogist, 63, 203-204.

Baños, J.O., Amouric, M., De Fourquet, C., and Baronnet, A. (1983) Interlayering and interlayer slip in biotite as seen by HRTEM. American Mineralogist, 68, 754-758.

Baronnet, A. (1972) Growth mechanisms and polytypism in synthetic hydroxyl-bearing phlogopite, American Mineralogist, 57, 1272-1293.

Baronnet, A. (1973) Sur les origines des dislocations vis et des spirales de croissance dans les micas. Journal of Crystal Growth, 19, 193-198.

Baronnet, A. (1980) Polytypism in micas: A survey with emphasis on the crystal growth aspects. In Current Topics in Materials Science, Volume 5, E. Kaldis ed., North-Holland Publishing Company, Amsterdam, 447-548.

Baronnet, A. (1997) Equilibrium and kinetic processes for polytypes and polysome generation. In Modular Aspects of Minerals 1 EMU Notes in Mineralogy, Volume 1, S. Merlino ed., Eötvös University Press, Budapest, 119-152.

Baronnet, A. and Amouric, M. (1986). Growth spirals and complex polytypism in micas. II. Occurrence frequencies in synthetic species. Bullettin de Minéralogie, 109, 489 508 .

Baronnet, A. and Kang, Z.C. (1989) About the origin of mica polytypes. Phase Transitions, 16/17, 477-493.

Baronnet, A., Amouric, M., and Chabot, B. (1976) Mécanismes de croissance, polytypisme et polymorphisme de la muscovite hydroxylée synthétique. Joumal of Crystal Growth, 32, 37-59.

Barshad, I. and Kishk, F.M. (1968) Oxidation of ferrous iron in vermiculite and biotite alters fixation and replaceability of potassium. Science, 162, 1041-1402.

Bassett, W.A. (1960) Role of hydroxyl orientation in mica alteration. Bulletin of the Geological Society of America, 71, 449-456.
Bell, I.A. and Wilson, C.J.L. (1977) Growth defects in metamorphic biotite. Physics and Chemistry of Minerals, 2, $153-169$.

Bell, I.A. and Wilson, C.J.L. (1981) Deformation of biotite and muscovite: TEM microstructure and deformation model. Tectonophysics, 78, 201-228.

Bell, I.A. and Wilson, C.J.L. (1986) TEM observations of defects in biotite and their relationship to polytypism. Bullettin de Minéralogie, 109, 163-170.

Bell, I.A., Wilson, C.J.L., McLaren, A.C., and Etheridge, M.A. (1986) Kinks in micas: Role of dislocations and (001) cleavage. Tectonophysics, 127, 49-65.

Bigi, S. and Brigatti, M.F (1994) Crystal chemistry and microstructures of plutonic biotite. American Mineralogist, 79, 63-72.

Bloch, A.M., Zhukhlistov, A.P., and Zvyagin, B.B. (1990) Centrosymmetric and noncentrosymmetric one-layer polytypes of metasomatic sericites in the upper Devon of the Tuva Through. Abstracts of the 15th General IMA Meeting, Beijing, China, Volume 1, 297.

Bloss, F.D., Gibbs, G.V., and Cummings, D. (1963) Polymorphism and twinning in synthetic fluorophlogopite. Journal of Geology, 71, 537-547.

Borutskiy, B.Y., Soboleva, S.V., and Golovanova, T.I. (1987) Three-layered 3Tc biotite from the Khibiny pluton. Transactions (Doklady) SSSR Academy of Sciences: Earth Science Section, 294, 141-143.

Caslavsky, J.L. and Vedam, K. (1970) The study of dislocations in muscovite mica by X-ray transmission topography. Philosophical Magazine, 22, 255-268.

Catti, M., Ferraris, G., and Ivaldi, G. (1989) Thermal strain analysis in the crystal structure of muscovite at $700^{\circ} \mathrm{C}$. European Journal of Mineralogy, 1, 625-632.

Chisholm, J.E. (1975) Crystallographic shear in silicate structures. Surface Defects of Solids, 4, 126-151.

Christoffersen, R. and Kronenberg, A.K. (1993) Dislocation interactions in experimentally deformed biotite. Journal of Structural Geology, 15, 1077-1095.

Dahl, P.S. and Dorais, M.J. (1996) Influence of $F(O H)_{-1}$ substitution on the relative mechanical strength of rock-forming micas. Journal of Geophysical Research, 101, 11519 11524.

Dornberger-Schiff, K. (1959) On the nomenclature of the 80 plane groups in three dimensions, Acta Crystallographica, 12, 173.

Dornberger-Schiff, K., Backhaus, K.-O., and Durovič, S. (1982) Polytypism of micas: OD-interpretation, stacking symbols, symmetry relations. Clays and Clay Minerals, 30, 364-374.

Durovič, S. (1979) Desymmetrization of OD structures. Kristall und Technik, 14, 1047-1053.

Durovič, S., Weiss, Z., and Backhaus, K.-O. (1984) Polytypism of micas. II. Classification and abundance of MDO polytypes. Clays and Clay Minerals, 32, 454-474.

Etheridge, M.A. and Hobbs, B.E. (1974) Chemical and deformational controls on recrystallization of mica. Contributions to Mineralogy and Petrology, 43, 111-124.

Etheridge, M.A., Hobbs, B.E., and Paterson, M.S. (1973) Experimental deformation of single crystals of biotite. Contributions to Mineralogy and Petrology, 38, 21-36.

Farmer, V.C. and Wilson, M.J. (1970) Experimental conversion of biotite to hydroxybiotite. Nature, 226, 841-842.

Filippov, L.V., Lipovsky, Y.O., and Kapitanova, T.A. (1976) Potassic basaltoids of Central Mongolia and come problems of abyssal magma formation. Geokhimiya, 4, 475489. (in Russian).

Frank, FC. (1949) The influence of dislocations on crystal growth. Discussions of the Faraday Society, $\mathbf{5}, 48$. 
Frank, F.C. (1951a) The growth of carborundum; dislocations and polytypism. Philosophical Magazine, 42, 1014-1021.

Frank, F.C. (1951b) Capillary equilibria of dislocated crystals. Acta Crystallographica, 4, 497-501.

Guidotti, C.B. and Sassi, P.F. (1998) Petrogenic significance of Na-K white mica mineralogy: Recent advances for metamorphic rocks. European Journal of Mineralogy, 10, 815854.

Guinier, A., Bokij, G.B., Boll-Dornberger, K., Cowley, J.M., Ďrovič, S., Jagodzinski, H., Khrisna, P., DeWolff, P.M., Zvyagin, B.B., Cox, D.E., Goodman, P., Hahn, Th., Kuchitsu, K., and Abrahams, S.C. (1984) Nomenclature of polytype structures. Report of the International Union of Crystallography Ad-Hoc Committee on the Nomenclature of Disordered, Modulated and Polytype Structures. Acta Crystallographica, A40, 399-404.

Güven, N. (1971) Structural factors controlling stacking sequences in dioctahedral micas. Clays and Clay Minerals, 19, 159-165.

Hartman, P. and Perdok, W.G. (1955) On the relations between structure and morphology of crystals, I. Acta Crystallographica, 8, 49-52.

Hendricks, S.B. and Jefferson, M.E. (1939) Polymorphism of the micas with optical measurements. American Mineralogist, 24, 729-771.

Ito, T. (1950) X-ray Studies on Polymorphism. Maruzen Co., Tokyo, $231 \mathrm{pp}$.

Juo, A.S.R. and White, J.L. (1969) Orientation of the dipole moments of hydroxyl groups in oxidized and unoxidized biotite. Science, 165, 804-805.

Kogure, T. (1997) On the structure of cleaved surfaces in biotite mica. Mineralogical Journal, 19, 155-164.

Kogure, T. and Nespolo, M. (1999a) A TEM study of longperiod mica polytypes: Determination of the stacking sequence of oxybiotite by means of atomic-resolution images and periodic intensity distribution (PID). Acta Crystallographica, B55, 507-516.

Kogure, T. and Nespolo, M. (1999b) First occurrence of a stacking sequence including $\left( \pm 60^{\circ}, 180^{\circ}\right)$ rotations in $\mathrm{Mg}$ rich annite. Clays and Clay Minerals, 47, 784-792.

Kogure, T. and Nespolo, M. (2001) Atomic structures of planar defects in oxybiotite. American Mineralogist, in press.

Kôzu, S. and Tsurumi, S. (1931) A consideration on chemical formula of anomite in basalt at Mutsure-jima. Journal of the Japanese Association of Minerologists, Petrologists and Economic Geologists, 5, 155-166. (in Japanese).

Kôzu, S. and Yoshiki, B. (1929) On biotite from Mutsurejima. Journal of the Japanese Association of Mineralogists, Petrologists and Economic Geologists, 1, 153-161. (in Japanese).

Kronenberg, A.K., Kirny, S.H., and Pinkston, J. (1990) Basal slip and mechanical anisotropy of biotite. Journal of Geophysical Research, 95, 19257-19278.

Lemmlein, G.G. and Dukova, E.D. (1956) Attraction between centers of spiral of opposite sign during crystal growth. Soviet Physics Crystallography, 1, 375-377.

Mares, V.M. and Kronenberg, A.K. (1993) Experimental deformation of muscovite. Journal of Structural Geology, 15, 1061-1075.

Nespolo, M. (1999) Analysis of family reflections of OD mica polytypes, and its application to twin identification. Mineralogical Journal, 21, 53-85.

Nespolo, M. and Takeda, H. (1999) Inhomogeneous mica polytypes: 8-layer polytype of the $2 M_{1}$ structural series determined by the periodic intensity distribution (PID) analysis of the X-ray diffraction pattern. Mineralogical Journal, 21, $103-118$
Nespolo, M., Takeda, H., and Ferraris, G. (1998) Represen tation of the axial settings of mica polytypes. Acta Crystallographica, A54, 348-356.

Nespolo, M., Takeda, H., Kogure, T., and Ferraris, G. (1999a) Periodic intensity distribution (PID) of mica polytypes: Symbols, structural model orientation and axial settings. Acta Crystallographica, A55, 659-676.

Nespolo, M., Kogure, T., and Ferraris, G. (1999b) Allotwinning: Oriented crystal association of polytypes compound. Some warning on consequences. Zeitschrift für Kristallographie, 214, 4-9.

Nespolo, M., Ferraris, G., Takeda, H., and Takéuchi, Y. (1999c) Plesiotwinning: Oriented crystal associations based on a large coincidence-site lattice. Zeitschrift für Kristallographie, 214, 378-382.

Nespolo, M., Ferraris, G., and Takeda, H. (2000) Identification of two allotwins of mica polytypes through the minimal rhombus unit in reciprocal space. Acta Crystallographica, B56, 639-647.

Noe, D.C. and Veblen, D.R. (1999) HRTEM analysis of dislocation cores and stacking faults in naturally deformed biotite crystals. American Mineralogist, 84, 1925-1931.

Ohta, T., Takeda, H., and Takéuchi, Y. (1982) Mica polytypism: Similarities in the crystal structures of coexisting $1 M$ and $2 M_{1}$ oxybiotite. American Mineralogist, 67, 298-310.

Pandey, D., Baronnet, A., and Krishna, P. (1982) Influence of the stacking faults on the growth of polytype structures in micas. Physics and Chemistry of Minerals, 8, 268-278.

Pauling, L. (1930) The structure of micas and related minerals. Proceedings of the National Academy of Sciences, 16, 123-129.

Peacock, M.A. and Ferguson, R.B. (1943) The morphology of muscovite in relation to the crystal lattice. University of Toronto Studies in Mineralogy, 48, 65-82.

Penn, R.L. and Banfield, J.F. (1998). Imperfect oriented attachment: Dislocation generation in defect-free nanocrystals. Science, 281, 969-971.

Ramsdell, L.S. (1947) Studies on silicon carbide. American Mineralogist, 32, 64-82.

Rieder, M. (1970) Lithium-iron micas from the Krušné hory Mountains (Erzgebirge): Twins, epitactic overgrowths and polytypes. Zeitschrift für Kristallographie, 132, 161-184.

Rieder, M., Huka, M., Kučerová, D., Minař́k, L., Obermajer, J., and Povondra, P. (1970) Chemical composition and physical properties of lithium-iron micas from the Krušné hory Mts. (Erzgebirge). Contributions to Mineralogy and Petrology, 27, 131-158.

Rieder, M., Cavazzini, G., D'yakonov, Yu. S., Frank-Kamenetskii, V.A., Gottardi, G., Guggenheim, S., Koval', P.V., Müller, G., Neiva, A.M.R., Radoslowich, E.W., Robert, J.L., Sassi, F.P., Takeda, H., Weiss, Z., and Wones, D.R. (1998) Nomenclature of the micas. Clays and Clay Minerals, 46, 586-595.

Ross, M., Takeda, H., and Wones, D.R. (1966) Mica polytypes: Systematic description and identification. Science, 151, 191-193.

Ross, M. and Wones, D.R. (1965) Polytypism in biotites. American Mineralogist, 50, 291.

Rule, A.C., Bailey, S.W., Livi, K.J.T., and Veblen, D.R. (1987) Complex stacking sequences in a lepidolite from Tørdal, Norway. American Mineralogist, 72, 1163-1169.

Sadanaga, R. and Takeda, H. (1968) Monoclinic diffraction patterns produced by certain triclinic crystals and diffraction enhancement of symmetry. Acta Crystallographica, B24, 144-149.

Sadanaga, R. and Takéuchi, Y. (1961) Polysynthetic twinning of micas. Zeitschrift für Kristallographie, 116, 406-429.

Silk, E.C.H. and Barnes, R.S. (1960) The Observation of Dislocations in Mica. United Kingdom Atomic Energy Au- 
thority Research Group Report, Metallurgy Division, Atomic Energy Research Establishment (AERE) R-3333. Harwell, Berkshire [a shorter version with the same title in Acta Metallurgica (1961) 9, 558-562].

Smith, J.V. and Yoder, H.S. (1956) Experimental and theoretical studies of the mica polymorphs. Mineralogical Magazine, 31, 209-235.

Smith, R.L., Bailey, R.A., and Ross, C.S. (1961) Structural Evolution of the Valles Caldera, New Mexico, and its Bear ing on the Emplacement of Ring Dikes. U.S. Geological Survey Professional Paper 366, 81 pp.

Sunagawa, I. (1964) Growth spirals on phlogopite crystals. American Mineralogist, 49, 1427-1434.

Sunagawa, I. (1977) Natural crystallization. Journal of Crystal Growth, 42, 214-223.

Sunagawa, I. (1978) Vapour growth and epitaxy of minerals and synthetic crystals. Journal of Crystal Growth, 45, 312.

Sunagawa, I. (1982) Morphology of crystals in relation to growth conditions. Estudios Geologique, 38, 127-134.

Sunagawa, I. and Bennema, P. (1982) Morphology of growth spirals, theoretical and experimental. In Preparation and Properties of Solid State Materials, Volume 7, Growth Mechanism of Silicon Nitrate, W.R. Wilcox, ed., Marcel Dekker Inc., New York, 1-129.

Sunagawa, 1. and Endo, Y. (1971) Morphology of nucleus of galena and phlogopite. Mineralogical Society of Japan Special Paper, 1, 25-29. (Proceeding IMA-IAGOD Meetings '70, IMA Volume).

Sunagawa, I. and Koshino, I. (1975) Growth spirals on kaolin group minerals. American Mineralogist, 60, 407-412.

Sunagawa, I. and Tomura, S. (1976) Twinning in phlogopite. American Mineralogist, 61, 939-943.

Sunagawa, I., Endo, Y., Daimon, N., and Tate, I. (1968) Nucleation, growth and polytypism of fluor-phlogopite from the vapour phase. Journal of Crystal Growth, 3,4, 751.

Sunagawa, I., Koshino, Y., Asakura, M., and Yamamoto, T, (1975) Growth mechanism of some clay minerals. Fortschritte der Mineralogie, 52, 217-224.

Takeda, H. (1967) Determination of the layer stacking se quence of a new complex mica polytype: A 4-layer lithium fluorophlogophite. Acta Crystallographica, 22, 845-853.

Takeda, H. and Donnay, J.D.H. (1966) Trioctahedral one-layer micas. III. Crystal structure of a synthetic lithium fluormica. Acta Crystallographica, 20, 638-646.

Takeda, H. and Morosin B. (1975) Comparison of observed and predicted parameters of mica at high temperature. Acta Crystallographica, B31, 2444-2452.

Takeda, H. and Ross, M. (1975) Mica polytypism: Dissimilarities in the crystal structures of coexisting $1 M$ and $2 M_{1}$ biotite. American Mineralogist, 60, 1030-1040.

Takeda, H. and Ross, M. (1995) Mica polytypism: Identification and origin. American Mineralogist, 80, 715-724.

Takeda, H., Haga, N., and Sadanaga, R. (1971) Structural investigation of polymorphic transition between $2 \mathrm{M}_{2^{-}}, 1 \mathrm{M}$ lepidolite and $2 M_{1}$ muscovite. Mineralogical Journal, 6 , 203-215.

Takéuchi, Y. (1997) Tropochemical Cell-Twinning. A Structure Building Mechanism in Crystalline Solids. Terra Scientific Publishing Company, Tokyo, 319 pp.
Takéuchi, Y. and Haga, N. (1971) Structural transformation of trioctahedral sheet silicates. Slip mechanism of octahedral sheets and polytypic changes of micas. Mineralogical Society of Japan Special Paper, 1, 74-87. (Proceedings IMA-IAGOD Meetings ' 70 , IMA Volume).

Tomisaka, T. (1958) On the chemical properties, optical properties and the structural types of some muscovites and phlogopites (Studies in the mica group, report 1). Journal of the Mineralogical Society of Japan, 3, 710-721. (in Japanese).

Tomisaka, T. (1962) Polytypes of the phlogopite-biotite series and their mutual relations. Journal of the Japanese Association of Mineralogists, Petrologists and Economic Geologists, 47, 134-143. (in Japanese, with English abstract).

Tomura, S., Kitamura, M., and Sunagawa, I. (1979) Surface microtopography of metamorphic white micas. Physics and Chemistry of Minerals, 5, 65-81.

Van Valkenburg, A. and Pike, R.G. (1952) Synthesis of mica. Journal of Research of the National Bureau of Standards, 48, 360-369.

Verma, A.R. (1953) Crystal Growth and Dislocations. Butterworths, London, $182 \mathrm{pp}$.

Wilson, C.J.L. and Bell, I.A. (1979) Deformation of biotite and muscovite: Optical microstructure. Tectonophysics, 58, $179-200$

Zhukhlistov, A.P. and Zyagin, B.B. (1991) The efficiency of electron diffraction in revealing $2: 1$ layer differing in structure and symmetry, found in dioctahedral micas and smectites. Proceedings of the 7th Euroclay, Conference, Dresden, 1211-1212.

Zhukhlistov, A.P., Zvyagin, B.B., and Pavlishin, V.I. (1990) Polytypic 4M modification of Ti-biotite with nonuniform alternation of layers, and its appearance in electron-diffraction patterns from textures. Soviet Physics Crystallography, 35, 232-236.

Zhukhlistov, A.P., Litsarev, M.A., and Fin'ko, V.I. (1993) First find of a six-layered triclinic $6 T c$ polytype of a Tioxybiotite. Transactions (Doklady) SSSR Academy of Sciences: Earth Science Section, 329, 188-194.

Zhukhlistov, A.P., Dragulescu, E.M., Rusinov, V.L., Kovalenker, V.A., Zvyagin, B.B., and Kuz'mina, O.V. (1996) Sericite with a non-centrosymmetric structure from the gold-silver base metal deposit Banská Štiavnica (Slovakia). Proceedings of the Russian Mineralogical Society, 125, 47-54. (in Russian, with English abstract).

Zvyagin, B.B. (1988) Polytypism of crystal structures. Computer Mathematics Applications, 16, 569-591.

Zvyagin, B.B. (1997) Modular analysis of crystal structures. In Modular Aspects of Minerals/EMU Notes in Mineralogy, Volume 1, S. Merlino ed., Eötvös University Press, Budapest, 345-372.

Zvyagin, B.B., Vrublevskaya, Z.V., Zhukhlistov, A.P., Sidorenko, O.V., Soboleva, S.V., and Fedotov, A.F. (1979) Highvoltage electron diffraction in the study of layered minerals. Nauka Press, Moscow, 224 pp. (in Russian).

Zvyagin, B.B., Rabotnov, V.T., Sidorenko, O.V., and Kotelnikov, D.D. (1985) Unique mica built of non-centrosymmetrical layers. Izvestia Akademyia Nauk SSSR (geology series) 5, 121-124.

E-mail of corresponding author: nespolo@nirim.go.jp (Received 7 March 2000; accepted 22 July 2000; Ms. 433; A.E. Stephen Guggenheim) 